\title{
Sustentabilidade e gestão de projetos: um estudo bibliométrico
}

\author{
Sandra Naomi Morioka ${ }^{\text {a* }}$, Marly Monteiro de Carvalho \\ anniversidade de São Paulo, São Paulo, SP, Brasil \\ *sandra.morioka@usp.br
}

\section{Resumo}

Os projetos conduzidos em empresas e organizações têm impactos no cenário imediato bem como em um futuro mais distante. Nesse contexto, o objetivo do trabalho é compreender a inserção de questões de sustentabilidade na literatura de gestão de projetos. A metodologia mescla técnicas de bibliometria, redes e análise de conteúdo. Os resultados apontam que as publicações estão dispersas em termos de autor e revista. As temáticas remetem a questões relacionadas aos stakeholders, inovação e desenvolvimento de produto, com destaque para publicações na área da construção civil. Observa-se uma tendência, principalmente a partir de 2005, com presença constante de artigos voltados ao pilar econômico e aumento da participação das abordagens social e ambiental. As pesquisas com o enfoque na tripla restrição - social, ambiental e econômica - tiveram nos últimos dois anos destaque no incremento. Há oportunidades de pesquisa significativas, já que as publicações estão longe de exaurir esse complexo tema.

\section{Palavras-chave}

Gestão de projetos. Sustentabilidade. Estudo bibliométrico.

\section{Introdução}

Recentemente, durante a execução de projetos, acidentes com expressivo impacto ambiental e social, por exemplo, nas áreas de petróleo, construção e infraestrutura de transporte, colocaram em evidência a necessidade de se tratar a questão da sustentabilidade de forma mais estruturada na área de gestão de projetos. A sustentabilidade tem ganhado evidência tanto no âmbito acadêmico quanto corporativo, podendo ser aplicada em diversos assuntos. Isso porque a sua lógica de inter-relacionamento entre os três pilares (social, ambiental e econômico) pode ser entendida como uma abordagem aplicável nos processos já existentes. Nesse contexto, a discussão sobre o desenvolvimento sustentável pode contribuir fortemente para a disciplina de gestão de projetos, aplicando a abordagem da tríade sociedade, economia e meio ambiente.

Sustentabilidade pode ser definida como o "[...] princípio que assegura que nossas ações de hoje não limitarão a gama de opções econômicas, sociais e ambientais disponíveis para as futuras gerações."
(Elkington, 2001, p. 21). No estudo de Dovers \& Hadmer (1992, p. 275), sustentabilidade é abordada da seguinte forma: "[...] habilidade de suportar ou de se adaptar a mudanças endógenas e exógenas de sistema humano, natural ou de ambos por tempo indefinido." Vale lembrar também que o significado primário do termo sustentabilidade, segundo o Dicionário Houaiss (Houaiss et al., 2009, p. 1866), é "característica ou condição do que é sustentável”, ou seja, daquilo que "é passivel de sustentação", que, por sua vez, é sinônimo de "conservação" e "manutenção". Assim, o uso do termo sustentabilidade pode acabar sendo empregado de diversas formas, tanto apoiado em definições acadêmicas, como as de Elkington (2001), Dovers \& Hadmer (1992) e Dovers (1995), como também no sentido mais básico de sua etimologia.

0 desenvolvimento sustentável é entendido por Elkington (2001, p. 21) da seguinte forma:

[...] é proposto pelos governos e líderes corporativos como solução para uma grande gama de problemas que agora estão começando a fazer parte da pauta internacional. 
Enquanto isso, Dovers \&t Hadmer (1992, p. 275) consideram que o desenvolvimento sustentável é "[...] o caminho para guiar mudanças e melhorias no sentido de manter ou melhorar a sustentabilidade do sistema." Dovers (1995), por sua vez, indica que a sustentabilidade é, portanto, um objetivo desafiador a ser perseguido, enquanto que o desenvolvimento sustentável é um processo variável em direção a esse objetivo. Dessa forma, verifica-se que os conceitos sustentabilidade e desenvolvimento sustentável não estão necessariamente alinhados entre as diversas publicações. Para fins do presente trabalho, adota-se o termo sustentabilidade que, à luz da amostra estudada, aponta para ambos os conceitos - sustentabilidade e desenvolvimento sustentável, por vezes sem uma clara definição que os distinga. A discussão desses conceitos à luz da gestão de projetos nesse contexto ainda é pouco madura para garantir uma distinção clara entre as definições dos termos.

0 presente trabalho tem como objetivo realizar uma revisão sistemática de literatura, buscando artigos que tenham abrangência em ambos os temas, tanto gestão de projetos quanto sustentabilidade. Busca-se identificar os principais assuntos tratados na interface dessas duas temáticas, identificando tendências e lacunas, bem como os principais autores e obras.

0 artigo está estruturado em quatro seções: a seção 2 descreve a metodologia de seleção da amostra de artigos a serem analisadas, bem como as etapas seguidas para própria análise dos dados. Na seção 3 são apresentados os resultados e a discussão da pesquisa, seguidos das conclusões, na seção 4 .

\section{Metodologia de pesquisa}

A abordagem metodológica adotada nesta revisão sistemática da literatura mesclas técnicas de bibliometria, redes e análise de conteúdo. As técnicas de bibliometria permitiram estabelecer tendências em termos do número de publicações e citações ao longo do tempo, principais autores e obras, por meio de análise de citação/cocitações utilizadas como proxy de impacto na comunidade acadêmica (Diodato, 1994; Neely, 2005). A técnica de análise de conteúdo (Bardin, 2004) foi utilizada na codificação e análise das principais temáticas da pesquisa. No tratamento dos dados e confecção das análises adotaram-se os softwares Sitkis (Schildt, 2002) e Ucinet (Borgatti et al., 2002).

A análise dos dados foi feita em três etapas. $\mathrm{Na}$ primeira etapa, os dados primários da amostra foram tratados, buscando-se ter uma visão quantitativa das publicações, tratando-se os dados e agrupando-os por ano de publicação, revista de publicação, número de citações, entre outros. Dado que o próprio banco de dados fornece as informações necessárias para tais análises de forma razoável, a sua execução foi possivel por meio de um tratamento em planilhas eletrônicas.

Já na segunda etapa da análise dos dados foi utilizado o software de apoio para tratamento de dados bibliométricos chamado Sitkis. Com licença gratuita, o programa é uma ferramenta baseada em Java que permite a elaboração de redes entre publicações de uma determinada amostra, bem como entre os trabalhos citados por essa amostra inicial. Assim, é possível identificar eventuais clusters de áreas afins e tirar conclusões interessantes do resultado gráfico possibilitado pelos programas associados Ucinet e Netdraw. Por meio desses resultados gráficos de ligação entre as unidades da amostra é possível identificar as publicações mais relevantes e como elas se inter-relacionam. Para tanto, utilizaram-se as redes de citação, cocitações e palavras-chave. Índices de centralidade e intermediação foram calculados para apoiar na interpretação das redes resultantes. As fórmulas correspondentes a esses índices são apresentadas na Tabela 1. 0 resultado dos índices normalizados de centralidade e de intermediação obtidos pela ferramenta Ucinet são apresentados na discussão dos resultados.

A última etapa de tratamento dos dados está baseada na análise de conteúdo dos artigos da amostra. Assim, por meio da leitura dos resumos e, eventualmente, do artigo na íntegra, foi feita a classificação entre econômico, ambiental e social, conforme o conceito do triple bottom line de Elkington (2001). É evidente que esses assuntos se entrelaçam de forma significativa, porém houve um esforço para identificar o pilar predominante de cada artigo. Para complementar essa análise de conteúdo, foram

Tabela 1. Formulação matemática dos índices de centralidade e intermediação.

\begin{tabular}{|c|c|c|}
\hline Centralidade & $C^{\prime} D(n i)=d(n i) /(g-1)$ & $\begin{array}{c}\text { C'D (ni) } \rightarrow \text { Centralidade } \\
\text { d (ni) } \rightarrow n^{\circ} \text { laços } \\
g \rightarrow n^{\circ} \text { atores }\end{array}$ \\
\hline Intermediação & $\operatorname{Pp}($ ni) $=($ li $/(g-1) / \Sigma D(n j, n i) / l i$ & 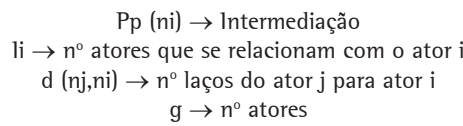 \\
\hline
\end{tabular}

Fonte: Wasserman \& Faust (1994). 
identificados artigos da amostra que têm no título, no resumo ou como palavra-chave os termos framework e/ou modelo. Apesar de não serem sinônimos, a proximidade de emprego desses termos permite que os artigos filtrados tragam propostas estruturadas para a sustentabilidade no contexto de gestão de projetos. Assim, são discutidos construtos e diagramas conceituais apresentados em seis artigos filtrados com esse critério.

\subsection{Amostra e procedimento de busca}

A amostra inicial foi obtida a partir de buscas na base ISI Web of Knowledge (Web of Science). Isso porque sua abrangência é bastante significativa, uma vez que nela é possível localizar informações de todos os periódicos indexados, cujos índices são apurados pelo Journal Citation Report (JCR), independentemente de sua base de origem. Além disso, essa base é capaz de fornecer dados de forma a tratar não somente a amostra inicial como também as suas referências, permitindo uma análise mais aprofundada das informações extraídas do banco de dados.

Em junho de 2010, a amostra inicial foi obtida a partir do primeiro filtro com a palavra sustain*, buscando identificar artigos que pudessem conter assuntos tanto de sustentabilidade quanto de desenvolvimento sustentável e suas variantes. Conforme discutido na seção introdutória, o termo sustentabilidade pode ser empregado nos artigos da amostra tanto apoiado em definições acadêmicas (Elkington, 2001; Dovers \& Hadmer, 1992; Dovers, 1995) como no sentido mais básico de sua etimologia, em artigos mais exploratórios. Portanto, dada a característica exploratória do tema, ainda pouco maduro, empregou-se apenas o termo sustentabilidade nas análises. Essa primeira etapa resultou em um grande número de artigos, mais de 440 mil títulos. Restringindo essas publicações com um novo filtro, project management, o banco de dados identificou 200 textos, contemplando os diversos tipos de publicação. Assim, a amostra utilizada pela presente pesquisa foi obtida, finalmente, selecionando-se somente as publicações do tipo artigo. Dessa forma, o número de títulos contidos na amostra utilizada para análise chegou a 102 artigos. A partir da amostra inicial, a amostra é ampliada para as referências dessa amostra inicial, pelo método bola de neve, compreendendo 2.761 referências, que serão tratadas nas redes de citação, artigos para referência, cocitações e palavraschave, já mencionadas.

\section{Apresentação e discussão dos resultados}

A seção a seguir tem como objetivo discutir os resultados das análises conduzidas a partir da amostra obtida, para que seja possível ter-se uma visão geral das publicações relativas a gestão de projetos e sustentabilidade.

\subsection{Análise inicial}

Observando-se o número de artigos publicados que tratam do tema gestão de projetos associado de alguma forma a sustentabilidade, verifica-se a presença de títulos desde 1991. Vale ressaltar que há um evidente crescimento desde 2005, com destaque para 2010 e 2011 (Figura 1).

Além disso, observando-se os dados obtidos é possível ver que os trabalhos da amostra estudada apresentam dispersão bastante considerável, podendo-se verificar isso principalmente na análise do número de artigos por autor e por revista, conforme mostrado pelas Figuras 2 e 3 . 1sso porque as duas revistas que mais estiveram presentes na amostra, ambas da área de construção (Journal of Construction Engineering and Management $\mathrm{e}$

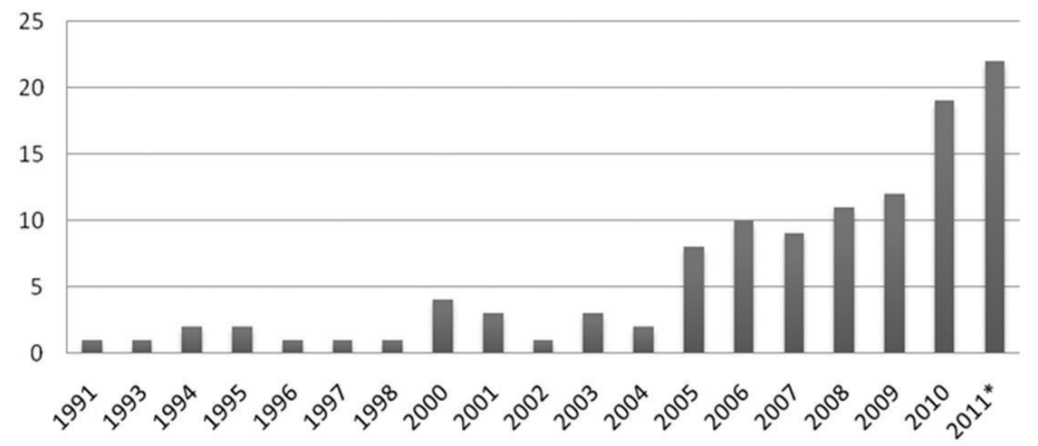

Figura 1. Evolução das publicações ao longo do tempo. Nota: *Estimado a partir das publicações até jun./2011. 


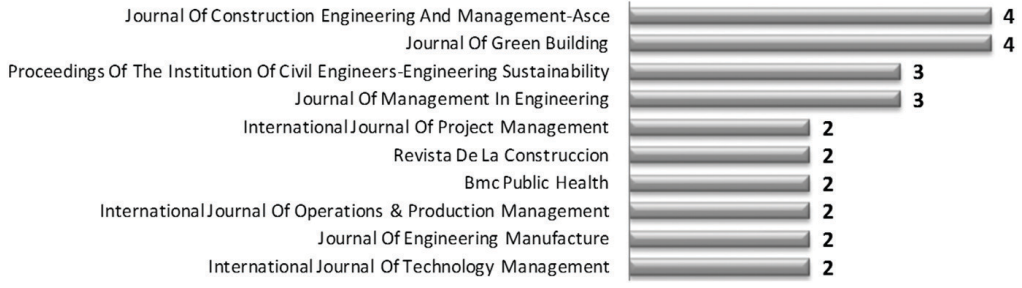

Figura 2. Gráfico de relevância das revistas da amostra.

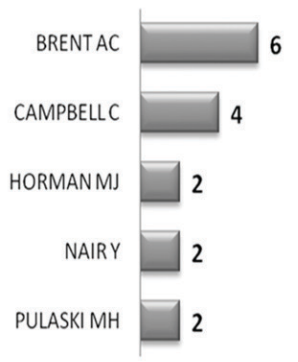

\begin{tabular}{|c|l|}
\hline $\begin{array}{c}\text { Principais } \\
\text { autores }\end{array}$ & \multicolumn{1}{|c|}{ Observação } \\
\hline $\begin{array}{c}\text { BRENT } \\
\text { (6 artigos) }\end{array}$ & $\begin{array}{l}\text { Enfoque em meio ambiente e } \\
\text { projetos de grande impacto } \\
\text { ambiental (ex energia e } \\
\text { agricultura) }\end{array}$ \\
\hline CAMPBELL & $\begin{array}{l}\text { Envolvimento da comunidade } \\
\text { na tomada de decisão, } \\
\text { principalmente na área da } \\
\text { saúde }\end{array}$ \\
\hline
\end{tabular}

Figura 3. Gráfico de relevância dos autores da amostra por número de artigos publicados.

Tabela 2. Os trabalhos mais citados.

\begin{tabular}{|c|c|c|c|}
\hline Autores & $\begin{array}{l}\text { Ano de } \\
\text { publicação }\end{array}$ & Principal abordagem & № citações \\
\hline Henard \& Szymanski & 2001 & $\begin{array}{l}\text { Inovação de produto para garantir o sucesso de longo prazo da } \\
\text { empresa }\end{array}$ & 217 \\
\hline Arora et al. & 2001 & $\begin{array}{l}\text { Características peculiares da Índia para garantir a perenidade do } \\
\text { sucesso da sua indústria de softwares }\end{array}$ & 73 \\
\hline Marais et al. & 2004 & Descrição de um projeto específico implantado na agricultura & 29 \\
\hline Williams et al. & 2003 & $\begin{array}{l}\text { Discussão sobre um framework focado em rupturas ou revisões } \\
\text { do projeto }\end{array}$ & 27 \\
\hline Naylor et al. & 2002 & $\begin{array}{l}\text { Descrição de um projeto na área da saúde, com enfoque na } \\
\text { participação da comunidade }\end{array}$ & 25 \\
\hline Reed & 2005 & $\begin{array}{l}\text { Relevância do envolvimento das pessoas de campo para que a } \\
\text { pesquisa acadêmica tenha relevância e aderência na área da saúde }\end{array}$ & 12 \\
\hline
\end{tabular}

Journal of Green Building), possuem apenas quatro artigos associados. No que concerne aos autores, apenas dois autores da amostra possuem mais do que duas publicações: Brent, com seis artigos, tem uma visão que prioriza a perspectiva ambiental, enquanto Campbell, notadamente, tem por foco fenômenos sociais (grassroots), em especial no setor de saúde.

Considerando os artigos da base de dados ISI Web of Science, as referências da amostra analisada que foram mais citadas são mostrados na Tabela 2. Os trabalhos tratam da sustentabilidade principalmente no sentido de garantir a perenidade da organização, não necessariamente considerando o inter-relacionamento entre as esferas econômica, social e ambiental no contexto da gestão de projetos. Observa-se que pelo critério de citação destacam-se os artigos de Henard
\& Szymanski (2001), com 217 citações, seguidos pelos de Arora et al. (2001), com 73 citações - ambos focam o conceito de sustentabilidade como longevidade, sucesso no longo prazo, priorizando a perspectiva econômica.

Assim, a partir dessas análises iniciais é possível chegar à conclusão parcial de que as pesquisas na interface entre gestão de projetos e sustentabilidade ainda estão bastante dispersas, tanto no que concerne aos veículos de publicação como em relação aos autores e obras de referência. Vale destacar que os principais trabalhos, considerando-se os critérios de citação e número de artigos publicados, não debateram especificamente a inserção de práticas (processos, ferramentas e métodos) de sustentabilidade na gestão de projetos. 


\subsection{Análise de redes}

Para uma visão da amostra e seus pilares teóricos, foram realizadas duas análises de rede, a primeira de artigos para referências e a segunda de cocitações.

A rede de artigos para referência vincula os artigos mais citados na amostra inicial e suas referências mais citadas. Adotou-se como critério de corte que as referências fossem citadas pelo menos duas vezes no texto e os artigos da amostra inicial que tinham pelo menos duas citações, garantindo uma maior aderência entre os trabalhos. A Figura 4 ilustra o resultado, no qual é possível observar que a amostra não apresentou nenhum agrupamento claro, já que a rede obtida está bastante dispersa, com vários pares ou trios isolados à esquerda da rede, despontando à direita um grupo mais conectado. Verifica-se ainda que não há obras que sejam referenciadas por mais do que três artigos da amostra inicial, o que indica que não há referências que tenham influência marcante sobre a amostra estudada. Destaca-se também que os trabalhos mais referenciados dentro da amostra foram os de Burns \& Stalker (1961) e Porter (1985), que além de serem mais antigos, não se referem diretamente à sustentabilidade, tratando de assuntos de cunho mais específico de gestão de inovação e estratégia competitiva, respectivamente. A referência de Porter (1985) pode ser um indicativo da relevância de se tratar da sustentabilidade com uma abordagem mais estratégica, como uma vantagem competitiva. A referência de Porter (1985) foi utilizada, por exemplo, por Betts (1994), que apresenta um estudo de caso em uma média empresa do setor de construção civil, no qual foi acompanhado o processo de planejamento estratégico aplicando-se conceitos de vantagem competitiva de longo prazo.

A Figura 5 traz o resultado da análise de cocitações dos artigos da amostra obtida. Esse gráfico permite identificar padrões de similaridade nas referências da amostra estudada. Mais uma vez estão presentes trabalhos de viés de estratégia, como os de Porter (1985), de Prahalad \& Hamel (1990) e de Betts \& Ofori (1992), reforçando a relevância do tema ao se tratar de assuntos relacionados à sustentabilidade. Para compreender melhor as relações entre as referências que se destacam como pilar teórico da amostra estudada foram calculados os índices de centralidade e intermediação, conforme ilustra a Tabela 1. Com base na análise das conexões, foram propostos agrupamentos (clusters) entre as referências. Observa-se na rede de cocitações três clusters principais: um central denominado estratégia, que destaca os trabalhos de Porter (1985) e de Prahalad \& Hamel (1990), utilizados nos artigos para fundamentar aspectos relacionados com vantagem competitiva de longo prazo e formação de competências estratégicas. 0 cluster da esquerda, denominado gestão, trata de obras clássicas que abordam o modelo japonês de produção (lean production), notadamente os trabalhos de Womack et al. (1990) e Ohno (1988), enquanto o trabalho de Hofstede (1980) aborda diferenças culturais. 0 cluster denominado construção civil é

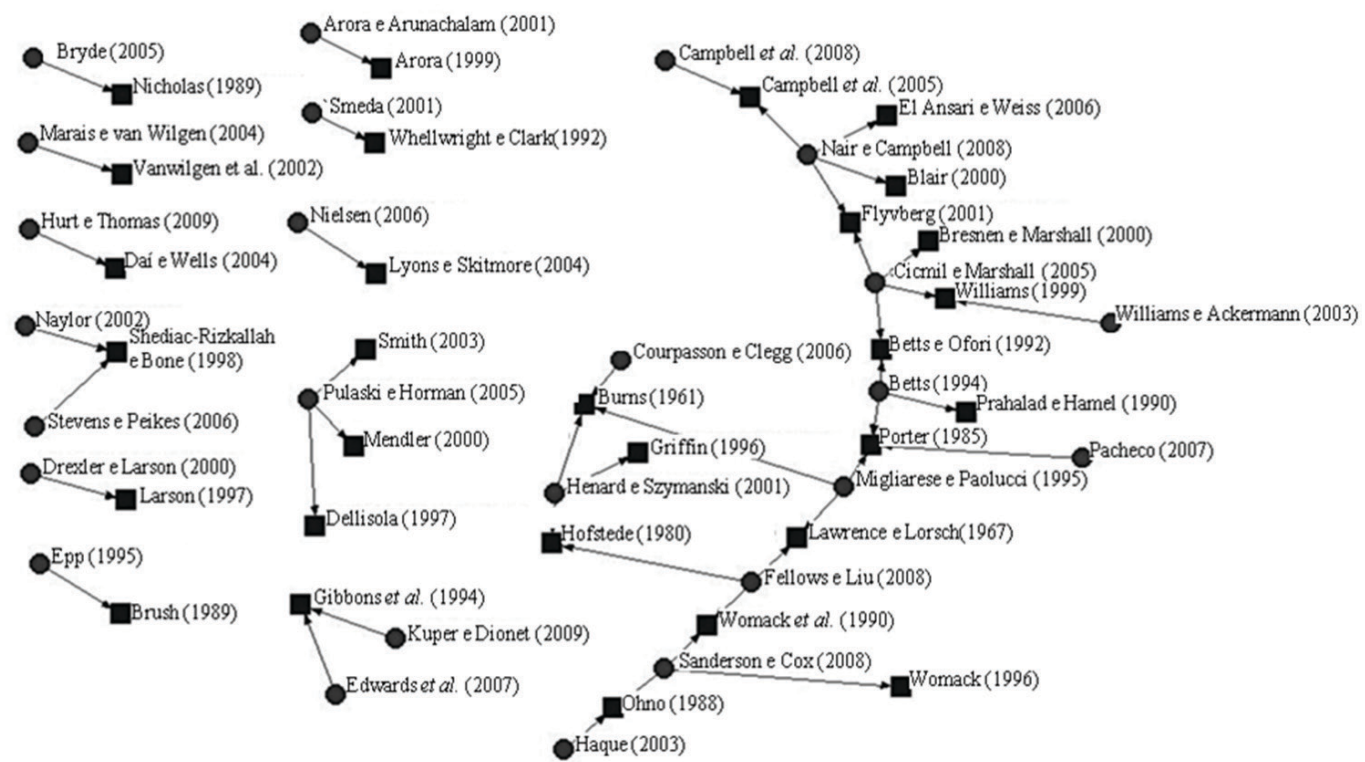

Figura 4. Rede de relacionamento entre artigos da amostra e suas referências. Nota: Gráfico feito no software Ucinet, com os dados importados pelo software Sitkis. Nota 1: Os quadrados representam as referências e os círculos representam os artigos. 


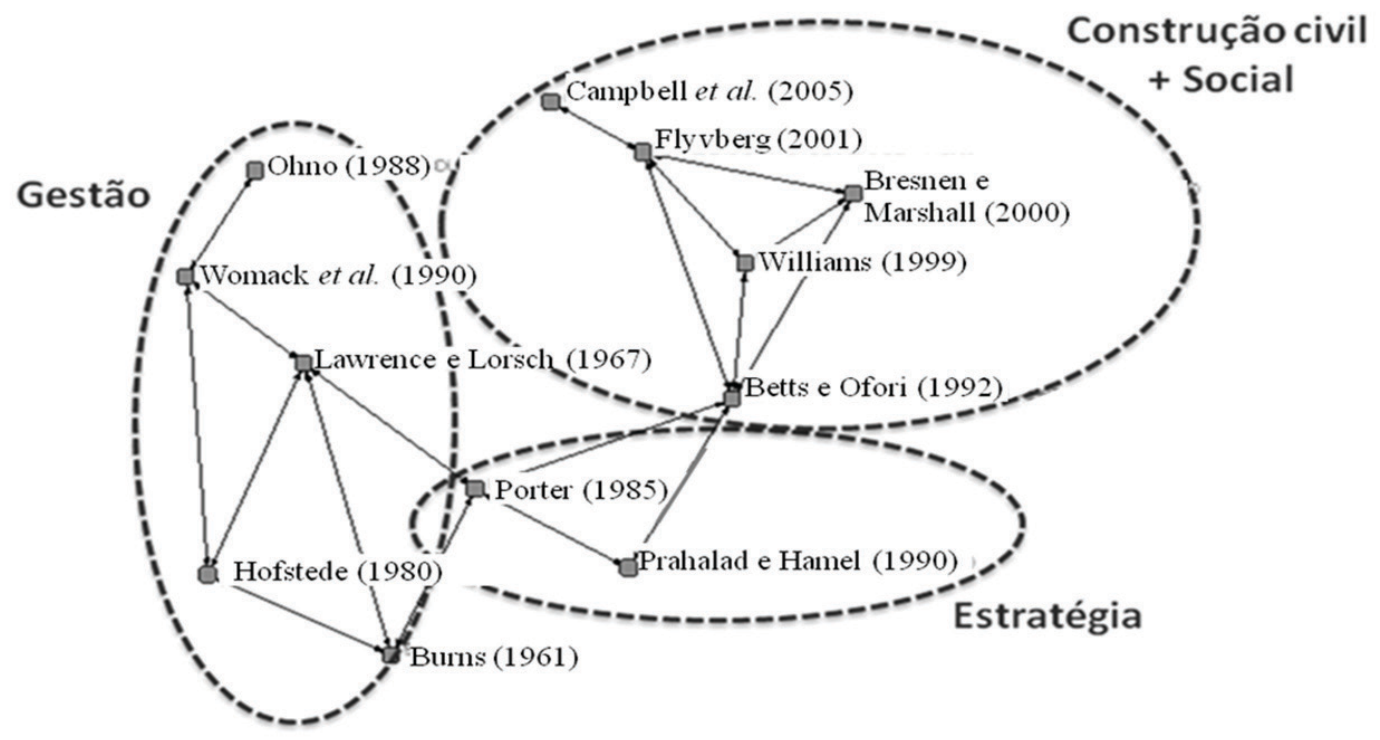

Figura 5. Análise de cocitações.

o que aborda temas mais próximos ao escopo dessa pesquisa, neles há aspectos de sustentabilidade incorporados na gestão de projetos de construção civil (ver Figura 5). Nesse último cluster observa-se uma tendência crescente de evolução em termos de sustentabilidade em projetos de construção - existe um conjunto de certificações com enfoque de empreendimentos sustentáveis.

Analisando-se a Tabela 3 verifica-se que os trabalhos de Betts \& Ofori (1992), Bresnen \& Marshall (2000) e Porter (1985) têm maior grau de centralidade inbound, i.e., o maior número de conexões para dentro, dessa forma, esses trabalhos são os mais proeminentes, podendo ser caracterizados como arquétipo de estrelas (stars) da rede (ver Tabela 3). Por outro lado, o índice de intermediação (nBetweenness) permite observar que também os trabalhos de Betts \& Ofori (1992) e Porter (1985), com índices mais elevados, assumem o papel de intermediadores entre os clusters, conectando os cluster gestão e construção, como apresentado na rede da Figura 5. Observa-se ainda que sem esses trabalhos a rede estaria desconectada (cutpoint), com 2 clusters isolados. Entre os trabalhos de maior centralidade vale destacar o livro de Lawrence \& Lorsch (1967), que trata de questões ambientais (environment), mas no contexto de ambiente competitivo em que analisa as incertezas. No entanto, já colocam o conflito de valor entre a ênfase no bem-estar das pessoas, em uma organização, ou a ênfase no desenvolvimento e sucesso da organização.

Observando-se as redes das palavras-chave é possivel identificar-se dois agrupamentos mais intensos, ilustrados pela espessura das linhas conectoras da
Tabela 3. Índices de centralidade e intermediação.

\begin{tabular}{lc}
\hline & $\begin{array}{c}\text { indice de centralidade } \\
\text { (NrmlnDeg)* }\end{array}$ \\
\hline Betts \& Ofori (1992) & 21,875 \\
Bresnen \& Marshall (2000) & 18,75 \\
Porter (1985) & 15,625 \\
Flyvbjerg (2001) & 15,625 \\
Williams (1999) & 12,5 \\
Prahalad \& Hamel (1990) & 12,5 \\
Lawrence \& Lorsch (1967) & 12,5 \\
Hofstede (1980) & 9,375 \\
Womack et al. (1990) & 9,375 \\
Burns \& Stalker (1961) & 9,375 \\
Campbell et al. (2005) & 3,125 \\
Ohno (1988) & 3,125 \\
\hline & Indice de intermediação \\
(nBetweenness)***
\end{tabular}

*Referências que obtiveram índice de centralidade (Network Centralization - Indegree) superior a zero; **Referências que obtiveram indice de intermediação (nBetweenness) superior a zero; Nota: índices calculados no software Ucinet.

Figura 6. Por um lado, verifica-se uma temática mais relacionada aos stakeholders, evidenciando-se a relação entre liderança, participação de demais interessados no assunto em questão e avaliação de um determinado evento baseado na opinião dessas partes. Ligado a esse grupo de nós, verificam-se 


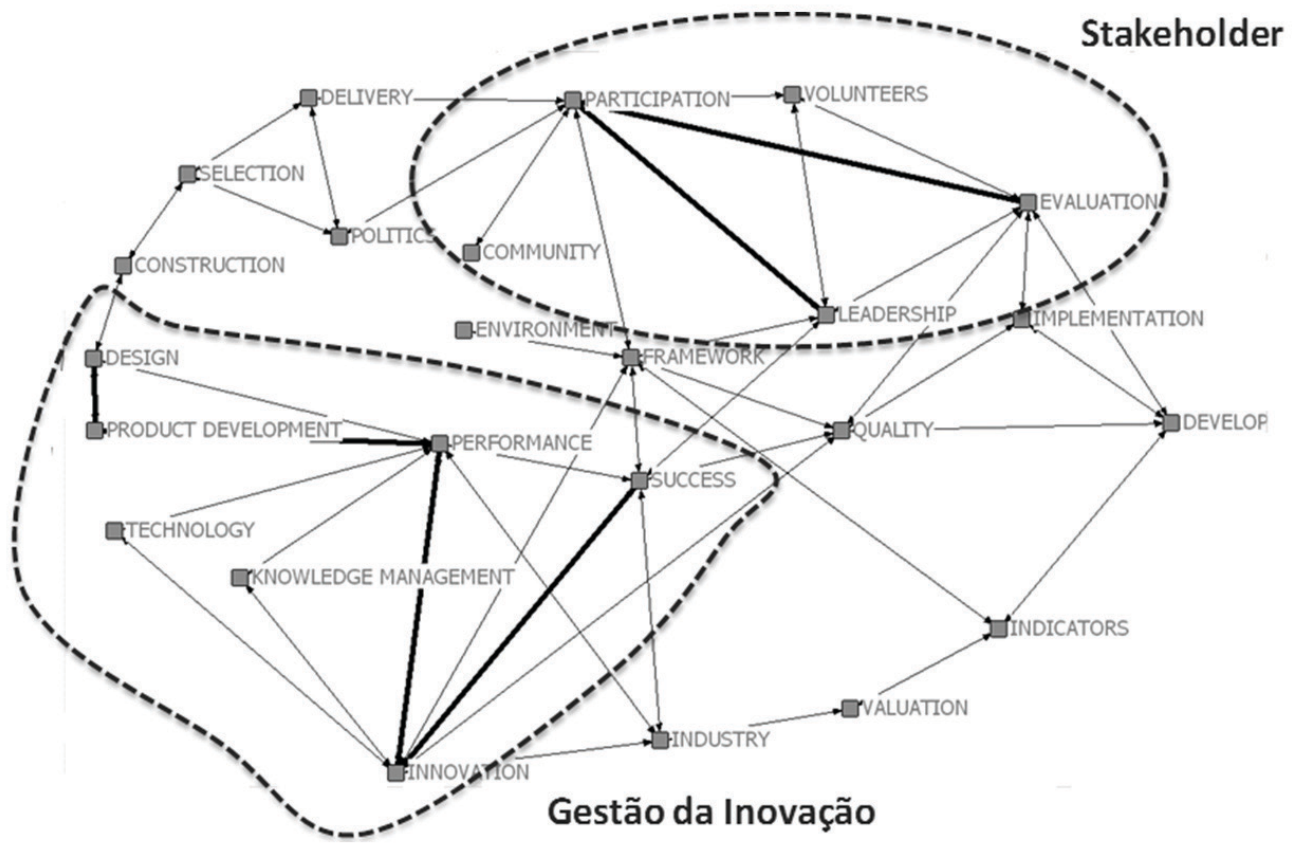

Figura 6. Análise de relacionamento das palavras-chave. Nota: A espessura das linhas representa a intensidade dos vínculos entre as palavras-chave, i.e., o número de vezes em que são citadas conjuntamente.

assuntos mais relacionados a diferentes grupos de interesse, tais como as iniciativas de voluntários, a comunidade e o poder público.

Por outro lado, verifica-se também a presença de uma vertente da inovação aliada à sustentabilidade, trazendo o relacionamento entre os conceitos sucesso e desempenho, principalmente para desenvolvimento de produto e para design (ver Figura 6). Ainda segundo a rede de palavras-chave (Figura 6), verifica-se que temas referentes à inovação e desempenho são também interconectados por intermédio de conceitos de tecnologia, bem como de gestão do conhecimento.

Sobre a Figura 6, ademais, pode-se dizer que chama a atenção o fato de as palavras construction e environment não estarem diretamente relacionadas. Assim, o tema construção, por sua ligação a design e, por conseguinte, a product development, não se refere necessariamente à construção civil (como Robichaud \& Anantatmula, 2011). Além disso, dado que o conceito de sustentabilidade mais moderno abrange os três pilares (econômico, social e ambiental), era de se esperar que a palavra environment pudesse estar diretamente ligada a temas como comunidade (ou sociedade). Porém isso não ocorre, sendo que essa ligação é intermediada por um tema que pode conter diversos assuntos: framework. Isso é um indicativo de que o conceito de triple bottom line (Elkington, 2001) não está sendo explorado de forma evidente na literatura.
Um dos principais conectores é a palavra-chave framework (ligada a seis outras palavras-chave), representando artigos que remetem à busca de estrutura e sistematização na elaboração de modelos conceituais. Esse nó tem relacionamento primário com diversos assuntos, tanto com os clusters já citados anteriormente de stakeholders e inovação, quanto com qualidade e indicadores. Uma análise crítica dos frameworks identificados na amostra de artigos será conduzida na seção 3.4. De um modo geral, verifica-se que a intersecção dos temas sustentabilidade e gestão de projetos ainda tem potencial significativo a ser explorado. Isso porque as delimitações entre agrupamentos de assuntos que poderiam tangenciar ambos os conceitos ainda não estão facilmente identificáveis. Além disso, não há trabalhos específicos que sejam utilizados por um número significativo de artigos, levando à conclusão que a dispersão verificada nos grafos pode ser justificada pelo fato de ainda não terem sidos publicados títulos de grande influência e relevância no âmbito acadêmico.

\subsection{Os pilares da sustentabilidade}

Conforme discutido inicialmente, a sustentabilidade considera três pilares: sociedade, economia e meio ambiente (Elkington, 2001). Esse assunto tem grande impacto na gestão de operações (Jiménez \& Lorente, 2001), sendo tratado em particular, no presente 
estudo, no contexto da gestão de projetos. Nesse sentido, os artigos da amostra foram classificados em uma das quatro perspectivas, tendo em vista a ênfase em cada um dos pilares da sustentabilidade ou no próprio triple bottom line, conforme ilustra a Tabela 4. Assim, as categorias encontradas são:

- Perpetuidade econômica: Artigos que enfocam a sustentabilidade como forma de manutenção e continuidade de existência, seja ela de uma determinada estratégia ou organização. De certa forma, essa visão de longo prazo, implícita ou explicitamente depende do texto, referindo-se à possibilidade econômica de manter-se perene.

- Pilar ambiental: Textos que enfatizam principalmente a questão ambiental, tanto em empresas de construção civil, para agregar valor à sua entrega final, quanto em um contexto de agricultura e de recursos naturais a serem geridos pelos governos.

- Pilar social: Buscando o bem-estar e a saúde de indivíduos, os artigos que tratam de gestão de projetos com ênfase em pessoas podem estar focados tanto em temas relativos a sociedades e comunidades quanto em discussões sobre a importância da abordagem de stakeholders internos a uma determinada organização.

- Triple bottom line: Nessa visão, os artigos tratam da sustentabilidade sob a óptica da integração dos três pilares da sustentabilidade.

Conforme ilustrado na Figura 7, é possível verificar que em trabalhos com participação significativa do setor público praticamente metade $(48 \%)$ focam principalmente o pilar social, enfatizando a importância da saúde da população, bem como seu bem-estar e educação. Já os artigos que têm como contexto o setor privado possuem predominância do tema sustentabilidade como sobrevivência econômica no longo prazo. Ainda nesse grupo de artigos verifica-se que a participação de artigos sobre o pilar ambiental e social é semelhante. Por um lado, os artigos com ênfase ambiental estão mais ligados ao setor de construção civil, tratando do desenvolvimento sustentável em seus projetos como forma de agregar valor a seus produtos perante o cliente. Por outro, o pilar social do setor privado dá mais atenção à necessidade da participação de diversos interessados no projeto, com o objetivo de aumentar as chances de sucesso, melhorar o desempenho etc.

Vale também ressaltar que um dos temas tratados pelos artigos que enfatizam o pilar social está relacionado à participação de diversos indivíduos envolvidos em um determinado projeto implantado. lsso pode ser verificado em diversos contextos, tanto na área da saúde (como em Naylor et al., 2002), quanto em um ambiente de gestão empresarial (como em Migliarese \& Paolucci, 1995). Assim, pode-se concluir que projetos e processos potencialmente se enriquecem com a participação de diversos pontos de vista, independentemente do contexto estudado.

Os dados indicam ainda que até a data de sua coleta a ênfase da perpetuidade econômica é bastante presente e constante. Esse pilar é então acompanhado por um aumento na produção de artigos sobre o tema gestão de projetos e sustentabilidade desde 2005 (ver Figura 8).

Tabela 4. Classificação dos artigos por perspectiva de sustentabilidade.

\begin{tabular}{|c|c|c|}
\hline Perspectiva & $\mathrm{N}^{0}$ referências & Referências \\
\hline Ambiental & 21 & $\begin{array}{l}\text { Thoma et al. (1993), Epp (1995), Eglington et al. (1998), Bernhardi et al. (2000), Marais et al. (2004), } \\
\text { Nijland (2005), Sell et al. (2006), Fourie \& Brent (2006), Mulder \& Brent (2006), Pulaski et al. (2006), } \\
\text { Weerasinghe et al. (2007), Steiner et al. (2008), Anning (2009), Van Berkel (2010), Wu \& Low (2010), } \\
\text { Molenaar et al. (2010), Nath \& lnoue (2010), Jaglin et al. (2011), Tsai et al. (2011), Ciomos et al. (2011), } \\
\text { Robichaud \& Anantatmula (2011) }\end{array}$ \\
\hline Econômica & 40 & $\begin{array}{l}\text { Sieli (1991), Betts (1994), Read \& Napier (1994), Nyamadi \& Yokogawa (1997), Drexler \& Larson } \\
\text { (2000), Divakar (2000), Wright (2000), Henard \& Szymanski (2001), Arora et al. (2001), Smeda (2001), } \\
\text { Williams et al. (2003), Haque (2003), Barry et al. (2003), Shore \& Cross (2004), Schuman \& Brent (2005), } \\
\text { Seitz et al. (2005), Courpasson \& Clegg (2006), Davidson et al. (2006), Nielsen (2006), Stevens \& Peikes } \\
\text { (2006), Pacheco-de-Almeida \& Zemsky (2007), Hirai et al. (2007), Thomas (2007), Frankel et al. (2008), } \\
\text { Nair \& Campbell (2008), Sanderson \& Cox (2008), Veas \& Pradena (2008), Salem \& Mohanty (2008), } \\
\text { Joham et al. (2009), Hurt \& Thomas (2009), Lee et al. (2009), Ustinovichius et al. (2010), Liu et al. (2010), } \\
\text { Petter et al. (2010), Bodea et al. (2010), Quante (2010), Olney et al. (2010), Chen (2011), Zahay et al. } \\
\text { (2011), Sengoku et al. (2011) }\end{array}$ \\
\hline Social & 30 & $\begin{array}{l}\text { Migliarese \& Paolucci (1995), Naylor et al. (2002), Reed (2005), Cicmil \& Marshall (2005), Bryde (2005), } \\
\text { Windel (2005), lsrar \& 1slam (2006), Edwards et al. (2007), Ottmann \& Street (2007), Mohammadi et al. } \\
\text { (2007), Gullick et al. (2007), Kakabadse et al. (2007), Campbell et al. (2008), Fellows \& Liu (2008), } \\
\text { McNulty et al. (2008), Leurs et al. (2008), Almansi \& Tammarazio (2008), Kuper et al. (2009), Barry et al. } \\
\text { (2009), Raven et al. (2009), Cust (2009), Bryde \& Leighton (2009), Couchman \& Fulop (2009), } \\
\text { Zhang et al. (2009), De Bruijne et al. (2010), Mewaldt (2010), Pavez et al. (2010), Mathews et al. (2010), } \\
\text { Madden \& Morawski (2011), Wesselink \& Wals (2011) }\end{array}$ \\
\hline $\begin{array}{l}\text { Tripple } \\
\text { bottom line }\end{array}$ & 11 & $\begin{array}{l}\text { Hartig et al. (1996), Pulaski \& Horman (2005), Jones (2006), Davies (2009), Fernandez-Sanchez \& } \\
\text { Rodriguez-Lopez (2010), Korkmaz et al. (2010), Corder et al. (2010), Ben Mahmoud-Jouini \& Lenfle } \\
\text { (2010), Turlea et al. (2010), Thomson et al. (2011), Laws \& Loeber (2011) }\end{array}$ \\
\hline
\end{tabular}




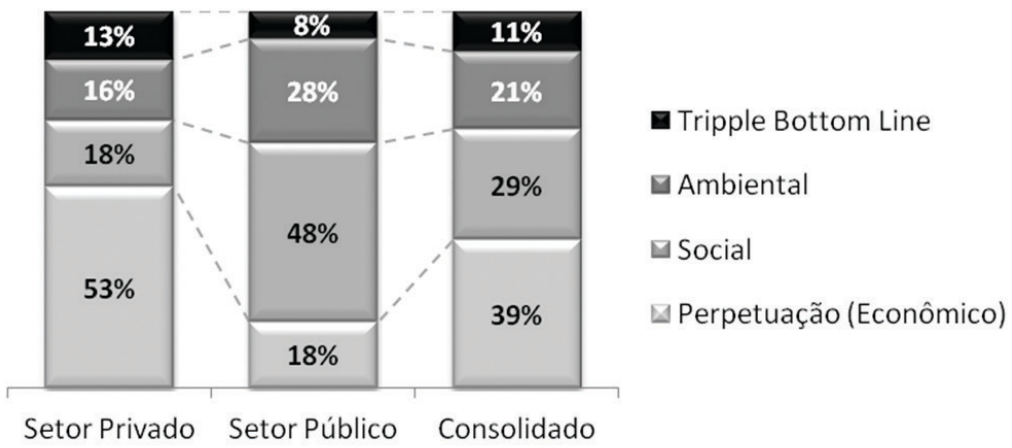

Figura 7. Distribuição dos artigos da amostra por pilar de sustentabilidade.

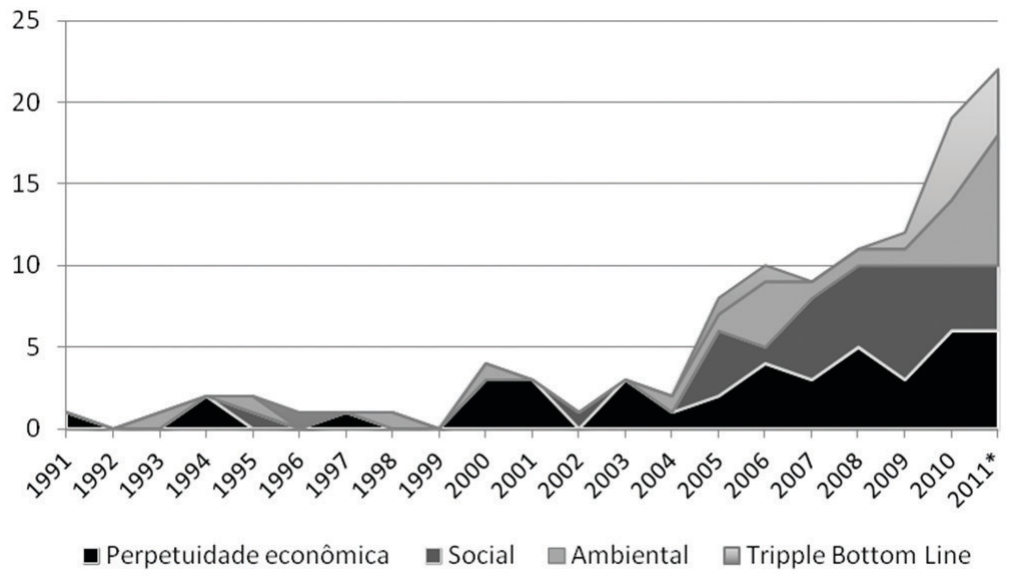

Figura 8. Comportamento dos pilares de sustentabilidade ao longo dos anos.

Observando a distribuição dos artigos que tiveram um dos pilares como ênfase, vale ressaltar que o conceito triple bottom line parece começar a se destacar apenas a partir de 2005 , tendo sofrido um impulso principalmente em 2010 e 2011. lsso indica que há uma tendência de que as novas publicações sobre sustentabilidade e gestão de projetos passem a abordar justamente dessa perspectiva tripla.

\section{Análise de frameworks e tabela-resumo}

Tomando como base a amostra de artigos retirada da base de dados ISI Web of Knowledge (Web of Science), seis artigos apresentaram os termos framework ou modelo no resumo ou como palavra-chave. Leurs et al. (2008), por exemplo, propõem o diagnóstico de colaboração sustentável denominado modelo DISC (Diagnosis of Sustainable Collaboration), que analisa evidências de colaboração entre organizações, comportamento organizacional e mudança organizacional planejada, buscando oportunidades para mudanças colaborativas. Nesse estudo de caso, os autores buscam validar os construtos propostos pelo modelo, a saber: fatores externos, contexto, gestão de mudança, gestão de projetos, apoio colaborativo (percepções, intenções e ações) e, finalmente, promoção coordenada da saúde (ver Figura 9). 0 modelo DISC apresenta maior evidência do pilar social triple bottom line. Além disso, apesar de não explicitamente, considera o termo sustentabilidade no sentido de perpetuação ao longo do tempo.

Um dos artigos inseridos no cluster da construção civil é o de Ustinovichius et al. (2010), que discute o uso de duas ferramentas de análise verbal na avaliação de risco de projetos de investimentos e escolha da empreiteira dada a incerteza das condições. Os autores discutem o resultado da aplicação de duas ferramentas, CLARA (Ustinovichius \& Kochin, 2003; Larichev et al., 2002) e Uni-ComBOS (Ashikhmin \& Furems, 2002). Um dos resultados da pesquisa foi a 


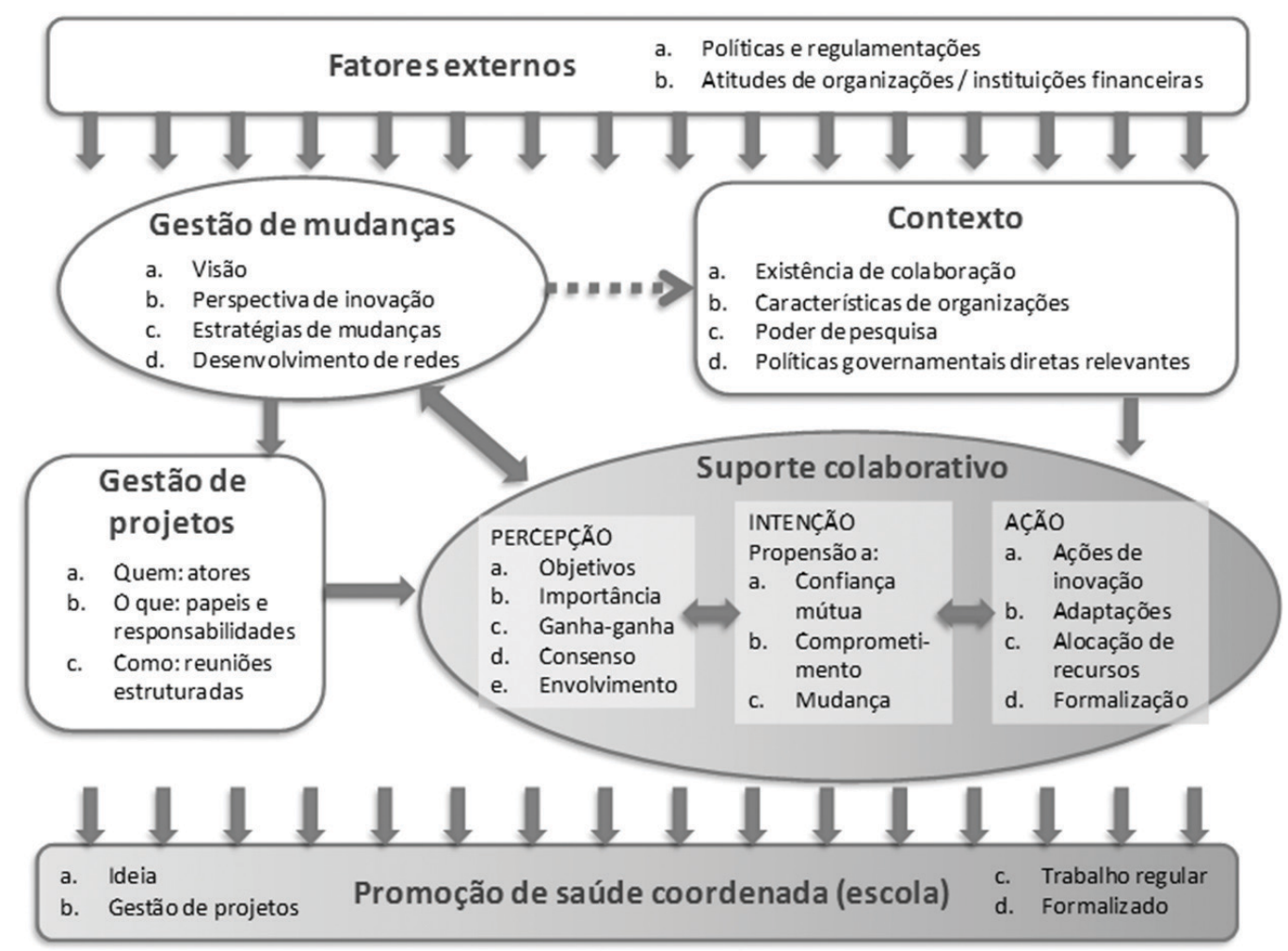

Figura 9. Modelo DISC (Diagnosis of Sustainable Collaboration). Fonte: Leurs et al. (2008).

identificação de categorias que interferem na tomada de decisão, baseada na análise de casos práticos de projetos de engenharia de multinacionais: especificação técnica, preço, termos de pagamento, garantias de desempenho, custo de seguro e limites de obrigações. Pode ser então verificado que critérios de análise de impactos socioambientais não são considerados pelos decisores pesquisados.

Também inserido no setor de construção, Fellows \& Liu (2008) discutem um framework para incorporar valores à gestão de projetos de construção, fazendo referência à sustentabilidade. Os autores destacam que os valores envolvidos no projeto determinam as variáveis de medição de desempenho, bem como a relativa importância de cada uma dessas variáveis. Os autores discutem a influência dos valores dos indivíduos sobre os projetos de construção com base no modelo apresentado por Liu (2006), que trata do desenvolvimento sustentável como composto por quatro sistemas: econômico, socioambiental, socioeconômico e legal. Os sistemas são caracterizados por dois fatores: curto/longo prazo e flexibilidade/ controle (Liu, 2006) (ver Figura 10).

Sánchez \& López (2010) focam o seu estudo em indicadores de projetos de construção, desenvolvendo uma metodologia para identificar, classificar e priorizar indicadores de sustentabilidade baseada em normas e publicações de gestão de riscos (ver Figura 11). Aplicando o método em um caso prático, os autores verificaram que a limitação de tempo e custo dificulta a execução do processo, sendo que as diversas técnicas de identificação de indicadores nem sempre apontam um mesmo resultado. Segundo os autores, o método mais adequado para entendimento da interrelação entre os indicadores é a entrevista.

Considerando a gestão de projetos em recursos hídricos, Bernhardi et al. (2000) buscam contribuir para a literatura propondo uma taxonomia para classificar métodos disponíveis. Dessa forma, os autores derivam um Modelo de Especificação de Gestão de Projetos (Project Management Specification Model - PMSM) para auxiliar na seleção, combinação e operacionalização de métodos, dependendo do tipo de projeto. Partindo de 56 métodos da literatura, os autores chegam a quatro classes de métodos básicos: (i) conceituação (comunicação, diagramação ou visualização); (ii) mensuração (coleta, geração ou questionamento); (iii) interpretação (votação, comparação ou evocação); e (iv) inferência (razão lógica, computação algébrica ou computação recursiva). Como ilustrado na Tabela 5, os autores propõem que 


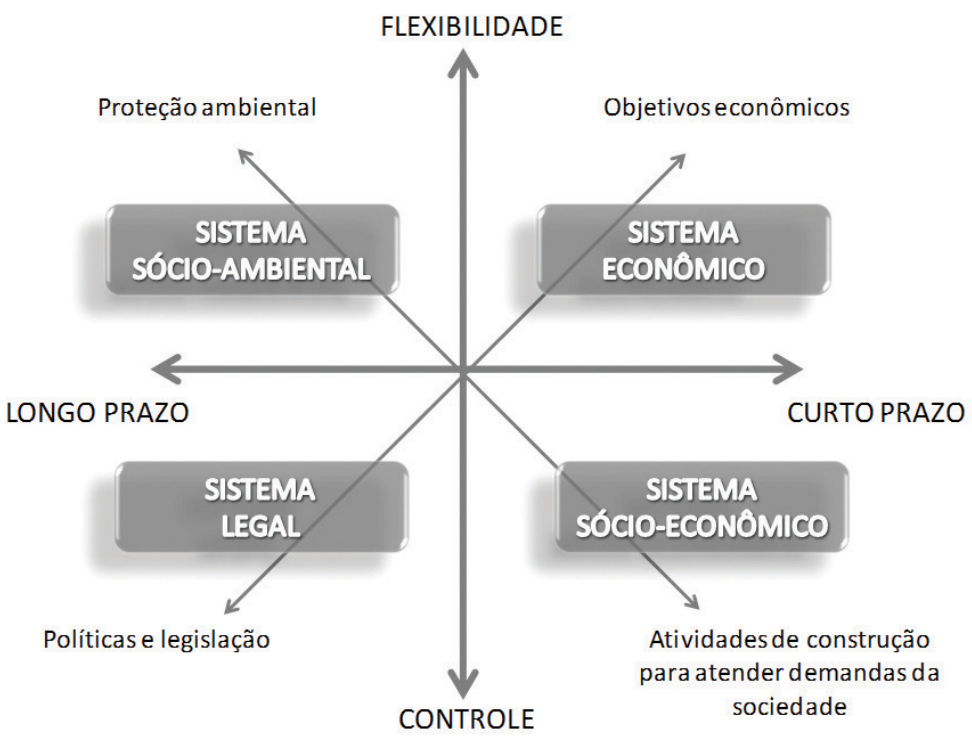

Figura 10. Sistemas do desenvolvimento sustentável. Fonte: Liu (2006).

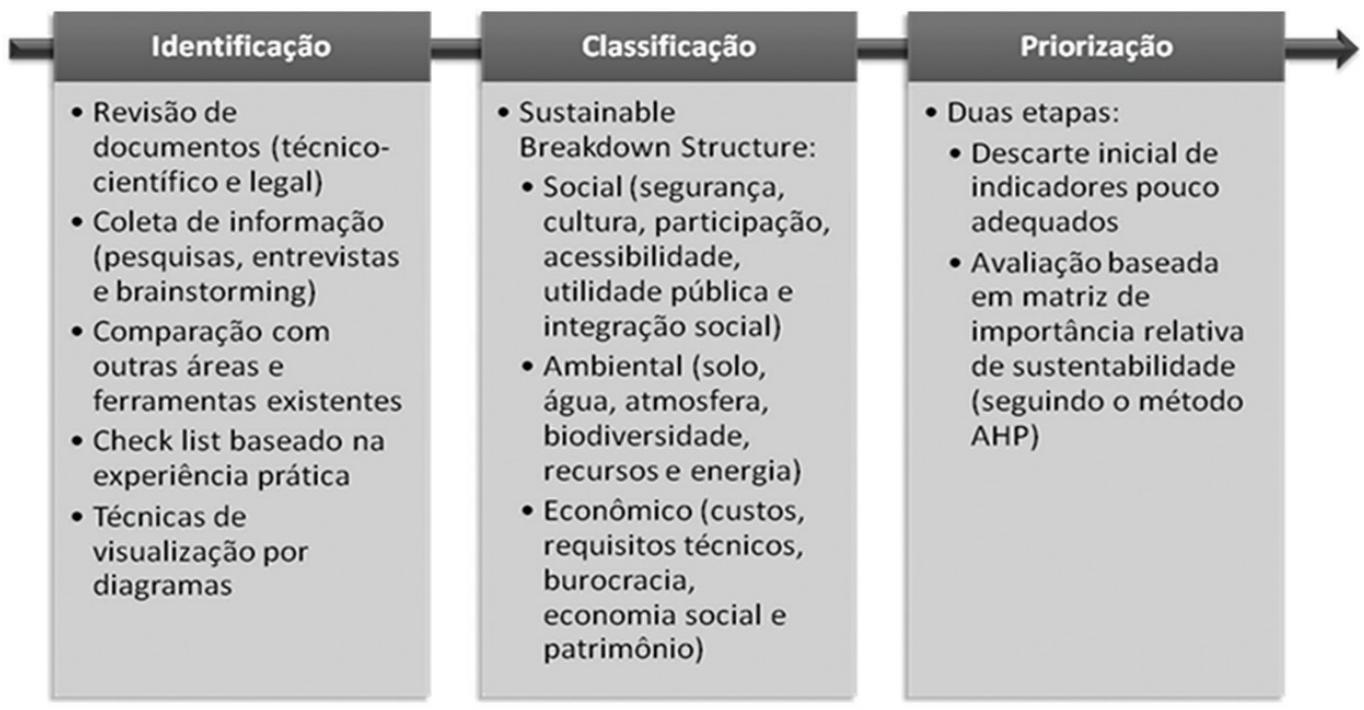

Figura 11. Etapas para identificar indicadores de sustentabilidade. Fonte: baseado em Sánchez \& López (2010).

Tabela 5. Critérios de escolha do método básico dada a característica do problema.

\begin{tabular}{lcccc}
\hline \multicolumn{1}{c}{ Critérios } & \multicolumn{2}{c}{ Classes de métodos básicos } \\
\hline $\begin{array}{l}\text { Complexidade } \\
\text { Incerteza }\end{array}$ & Conceituação & Mensuração & Interpretação & Inferência \\
$\begin{array}{l}\text { Certeza } \\
\text { Conhecimento limitado }\end{array}$ & $\mathrm{X}$ & $\mathrm{X}$ & $\mathrm{X}$ & $\mathrm{X}$ \\
$\begin{array}{l}\text { Conhecimento vasto } \\
\text { Grupo de especialistas }\end{array}$ & $\mathrm{X}$ & & $\mathrm{X}$ & $\mathrm{X}$ \\
$\begin{array}{l}\text { Indivíduo especialista } \\
\text { Fase inicial do projeto }\end{array}$ & $\mathrm{X}$ & $\mathrm{X}$ & $\mathrm{X}$ & \\
Sistema de tratamento de esgoto e água & $\mathrm{X}$ & $\mathrm{X}$ & $\mathrm{X}$ \\
Sistema de água urbano, regional, nacional & $\mathrm{X}$ & $\mathrm{X}$ & $\mathrm{X}$ & \\
\hline
\end{tabular}

Fonte: Bernhardi et al. (2000). 
a decisão quanto ao(s) método(s) a ser(em) utilizado(s) na prática seja realizada com base em critérios de decisão como grau de complexidade e incerteza. Assim, os atores do projeto, tais como consultores em gestão de recursos hídricos, engenheiros e gerentes de projeto, devem participar o quanto antes e o mais intensamente possível da seleção e uso dos métodos, garantindo adequação de escolha e consequente qualidade do projeto.

Tendo como objetivo incorporar princípios de desenvolvimento sustentável à gestão de projetos na área de recursos minerais, Corder et al. (2010) propõem o conceito SUSOP ${ }^{\circledR}$ (SUStainable OPerations). Baseados em iterações de casos práticos, o objetivo dos autores é desenvolver um mecanismo para: (i) gerar um projeto de fábrica viável e opções de operação de acordo com princípios de desenvolvimento sustentável; (ii) avaliar benefícios e impactos para essas opções; (iii) avaliar cada opção utilizando um processo de suporte à decisão baseado no desenvolvimento sustentável; e (iv) dar suporte à engenharia, à gestão de projetos e, consequentemente, a operações de projetos. A publicação de Corder et al. (2010) conduz as discussões iniciais sobre o SUSOP ${ }^{\circledR}$ oferecendo soluções concretas de forma limitada.

Um dos assuntos abordados é o processo para avaliar oportunidades de desenvolvimento sustentável tomando como fundamentação uma vasta base de conhecimento e composto pelas seguintes etapas: familiarização, definição de objetivo, identificação e avaliação (ver Figura 12). Os autores também destacam os principais fatores que influenciam na tomada de decisão: tarefas, orçamento/cronograma, padronizações e regulações (conformidade), entregáveis, indicadores (Key Performance Indicators - KPIs) e foco do projeto.

Inicialmente, vale ressaltar que as referências analisadas reforçam a evidência de que o uso do termo sustentabilidade e suas derivações não trazem as mesmas premissas. Os artigos com referência a framework ou model podem, por um lado, utilizar o conceito sustentabilidade como manutenção e continuidade (por exemplo, Leurs et al., 2008) e, por outro, empregar o termo no sentido de incorporar impactos ambientais e sociais às decisões até então tomadas a partir de paradigmas financeiros (Fellows \& Liu, 2008; Sánchez \& López, 2010; Corder et al., 2010). Além disso, chama a atenção os artigos não apresentarem uma solução concreta de como operacionalizar princípios de desenvolvimento sustentável nos processos de gestão de projetos. A publicação cujo objetivo de pesquisa esteve mais próximo a essa discussão foi a de Corder et al. (2010), porém mesmo essa deixa claro que há ainda muito campo a ser pesquisado na área de gestão de projetos alinhada ao desenvolvimento sustentável. A Tabela 6 traz um resumo esquemático dos frameworks discutidos até então.

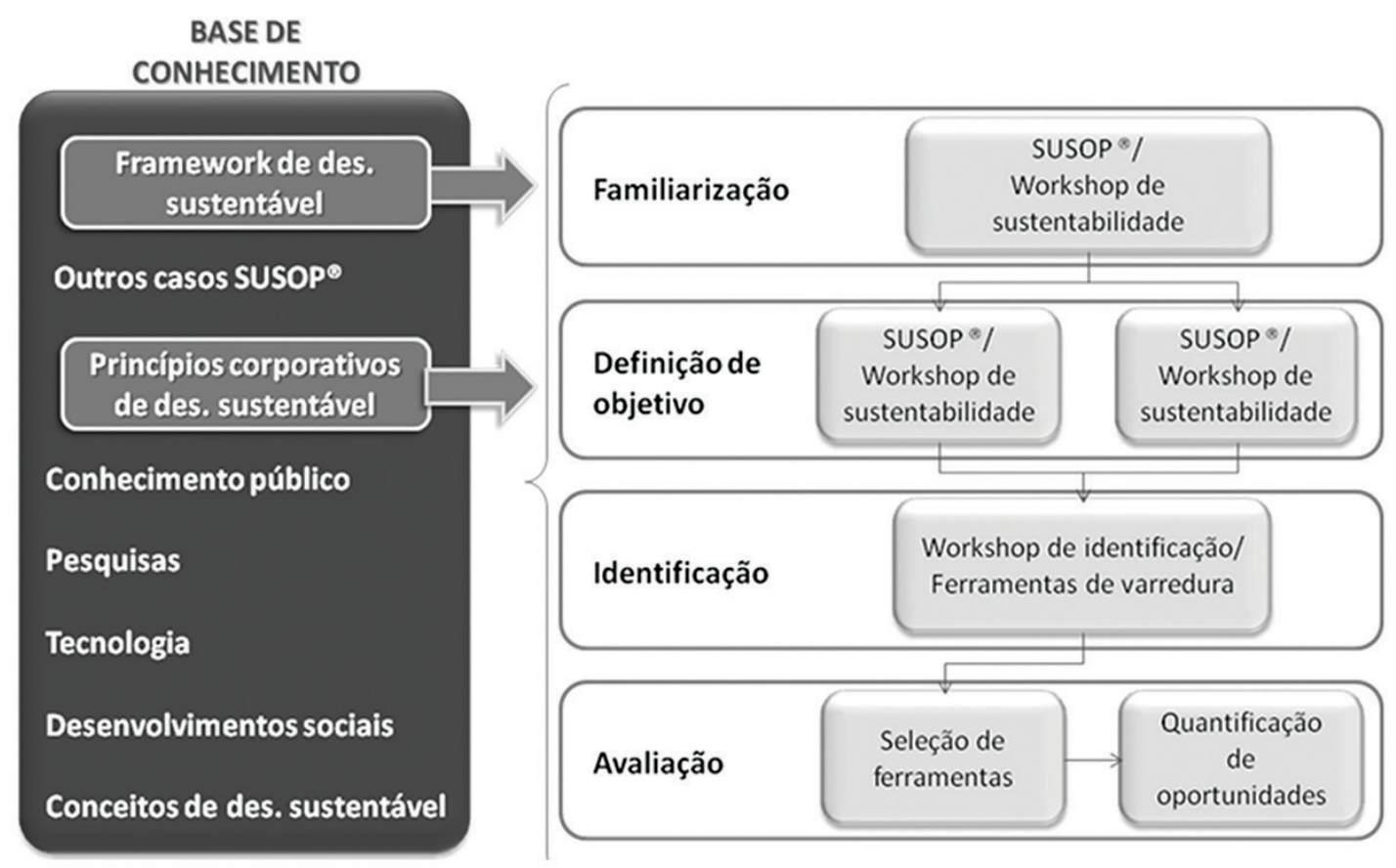

Figura 12. Processo de avaliação de oportunidades. Fonte: Corder et al. (2010). 
Tabela 6. Resumo dos frameworks identificados na amostra estudada.

\begin{tabular}{|c|c|c|c|c|}
\hline Ano & Autor & Objetivo do framework & Construtos associados & Setor \\
\hline 2010 & Ustinovichius et al. & $\begin{array}{l}\text { Métodos verbais de decisão em } \\
\text { investimentos }\end{array}$ & Métodos CLARA e ComBOS & Construção civil \\
\hline 2010 & Sánchez \& López & $\begin{array}{l}\text { ldentificação de indicadores de } \\
\text { sustentabilidade }\end{array}$ & $\begin{array}{l}\text { Etapas do processo: identificar, classificar e } \\
\text { priorizar }\end{array}$ & Construção civil \\
\hline 2010 & Corder et al. & SUSOP ${ }^{\circledR}$ (SUStainable OPerations) & $\begin{array}{l}\text { Processo para avaliar oportunidades de } \\
\text { desenvolvimento sustentável: familiarização, } \\
\text { definição de objetivo, identificação e avaliação }\end{array}$ & Recursos minerais \\
\hline 2008 & Fellows \& Liu & $\begin{array}{l}\text { Avaliação de projetos com foco em } \\
\text { sustentabilidade }\end{array}$ & $\begin{array}{l}\text { Quatro sistemas: econômico, socioambiental, } \\
\text { socioeconômico e legal, resultado da } \\
\text { combinação de dois fatores: curto prazo e } \\
\text { flexibilidade/controle }\end{array}$ & Construção civil \\
\hline 2008 & Leurs et al. & $\begin{array}{l}\text { Diagnóstico de colaboração } \\
\text { sustentável (CISC) }\end{array}$ & $\begin{array}{l}\text { Fatores relevantes: fatores externos, contexto, } \\
\text { gestão de mudança, gestão de projetos, apoio } \\
\text { colaborativo (percepções, intenções e ações) e } \\
\text { promoção coordenada da saúde }\end{array}$ & Saúde \\
\hline 2000 & Bernhardi et al. & $\begin{array}{l}\text { Taxonomia de métodos para apoio à } \\
\text { tomada de decisão }\end{array}$ & $\begin{array}{l}\text { Quatro classes de métodos básicos: } \\
\text { conceituação; mensuração, interpretação e } \\
\text { inferência }\end{array}$ & Recursos hídricos \\
\hline
\end{tabular}

\section{Conclusões}

0 trabalho teve como objetivo extrair uma visão geral sobre os principais artigos que tratam tanto de gestão de projetos quanto de sustentabilidade. Isso porque, uma vez que os projetos possuem consequências que vão além de seu término, o estudo de processos ligados à gestão de projetos pode ser uma ferramenta relevante para fazer com que as organizações utilizem abordagem sustentável. Assim, verificar as publicações existentes no assunto contribui fortemente na identificação de lacunas de conhecimento a serem preenchidas no futuro.

Observando os 102 artigos da amostra obtida pela busca na base de dados ISI Web of Knowledge (Web of Science), verifica-se que os artigos são recentes e ainda estão bastante dispersos, não podendo ser identificados nem autores e nem revistas com destaque nesse tipo de publicação. Além disso, os artigos mais relevantes em termos de citações tratam da sustentabilidade sob uma visão mais específica de um determinado pilar (social, econômico ou ambiental) e não de como a gestão de projetos se relaciona com o triple bottom line.

Ainda, algumas evidências apontam para um esforço maior para estudar o desenvolvimento sustentável em projetos na área da construção civil. Isso porque as revistas de maior concentração de publicações dentro da amostra estudada são dedicadas a essa área. Além disso, um importante cluster de publicações na área de construção civil foi identificado na análise de cocitações, bem como uma boa parte dos escassos frameworks encontrados na literatura dedica-se a esse setor.

Quando se fala em sustentabilidade tratada juntamente à gestão de projetos, diversos stakeholders devem ser envolvidos, tais como a comunidade e a liderança, evidenciando a importância da participação e parceria entre os envolvidos. Essa temática foi identificada principalmente analisando-se os agrupamentos da rede de palavras-chave e constitui uma relevante pauta para pesquisas futuras.

Assim, conforme mostrado neste trabalho, os artigos que tratam tanto de gestão de projetos quanto de sustentabilidade estão sendo publicados há mais de duas décadas, sendo que a partir de 2005 verifica-se um incremento no número de artigos. Nesse período, as publicações sobre sustentabilidade no contexto de perenidade econômica estiveram sempre presentes, de certa forma. Enquanto isso, o enfoque na abordagem tanto social e ambiental quanto do triple bottom line tem ganhado força, mais recentemente nos últimos seis anos, com grande tendência de crescimento. Nesse contexto, há bastante oportunidades para se explorar a gestão de projetos como forma de introduzir conceitos de sustentabilidade nas organizações. Portanto, demanda-se da comunidade acadêmica um esforço de pesquisa, dada a escassez de frameworks para operacionalizar o desenvolvimento sustentável à gestão de projetos. Estudos nessa área têm potencial para contribuir fortemente na literatura do assunto.

Uma implicação para a prática é que apesar de os frameworks de gestão de projetos estarem se difundindo, a questão da sustentabilidade no contexto de triple bottom line tem sido negligenciada também no dia a dia de projetos que adotam esses frames como referência. Para tanto, pesquisadores e praticantes podem em um primeiro momento apoiarem-se em metodologias e frameworks da área de gestão de projetos e adaptá-los para a lógica do desenvolvimento sustentável. Dessa forma é possível aproveitar o conhecimento de gestão de projetos 
já consolidado e construir conceitos e atributos incrementais de sustentabilidade, aproveitando, por exemplo, as áreas de conhecimento (integração, escopo, prazo, custo, qualidade, recursos humanos, comunicação, riscos e suprimentos) e os grupos de processos (inicialização, planejamento, execução, monitoramento/controle e finalização) do Project Management lnstitute - PMI (2008).

Este estudo apresenta limitações resultantes das escolhas metodológicas. A primeira relacionada à escolha da amostra inicial nas bases ISI Web of Science, em que se encontram os periódicos indexados pelo $J C R$ de várias bases de dados distintas, mas que têm um número limitado de títulos, o que pode ter deixado trabalhos relevantes fora da amostra estudada. Essa limitação é parcialmente mitigada pela adoção do método bola de neve, em que se amplia a pesquisa também para as referências dos artigos da amostra. Segundo, a análise de citação gera um viés temporal dado que os trabalhos e referências mais citadas tendem a ser os mais antigos.

\section{Referências}

Almansi, F., \& Tammarazio, A. (2008). Mobilizing projects in community organizations with a long-term perspective: neighbourhood credit funds in Buenos Aires, Argentina. Environment and Urbanization, 20(1), 121-147. http:// dx.doi.org/10.1177/0956247808089289

Anning, H. (2009). Case study: bond university mirvac school of sustainable development building, Gold Coast, Australia. Journal of Green Building, 4(4), 39-54. http:// dx.doi.org/10.3992/jgb.4.4.39

Arora, A., Arunachalam, V. S., Asundi, J., \& Ronald, F. (2001). The Indian software services industry. Research Policy, 30(8), 1267-1287. http://dx.doi.org/10.1016/ S0048-7333(00)00148-7

Ashikhmin, 1., \& Furems, E. (2002). UniComBOS: intelligent decision support system for multicriteria comparison and choice. Journal of Multi-Criteria Decision Analysis, $13(2-$ 3), 147-157.

Bardin, L. (2004). Análise de conteúdo. (3 ed.). Lisboa: Edições 70.

Barry, M. L., Steyn, H., \& Brent. A. C. (2009). Determining the most important factors for sustainable energy technology selection in africa. South African Journal of Industrial Engineering, 20(2), 33-51.

Barry, N., Campbell, P., Reed, N., Reid, M. E., Bower, D. J., Norrie, J., \& Currie, G. D. (2003). Implementation of videoconferencing to support a managed clinical network in Scotland: lessons learned during the first 18 months. Journal of Telemedicine and Telecare, 9(Suppl. 2), S7-S9. PMid:14728747. http:// dx.doi.org/10.1258/135763303322596110

Bernhardi, L., Beroggi, G. E. G., \& Moens, M. R. (2000). Sustainable water management through flexible method management. Water Resources Management, 14(6), 473495. http://dx.doi.org/10.1023/A:1011105008526

Betts, M. (1994). Sustainable competitive advantage for project-management consultants. Journal of
Management in Engineering, 10(1), 43-51. http://dx.doi. org/10.1061/(ASCE)9742-597X(1994)10:1(43)

Betts, M., \& Ofori, G. (1992). Strategic panning for competitive advantage in construction. Construction Management and Economics, 10(6), 511-532. http:// dx.doi.org/10.1080/01446199200000049

Bodea, C. N., Elmas, C., Tănăsescu, A., \& Dascălu, M. (2010). An ontological-based model for competences in sustainable development projects: a case study for project's commercial activities. Amfiteatru Economic, 12(27), 177-189.

Borgatti, S., Everentt, M., \& Freeman, L. (2002). Ucinet for Windows: software for social network analysis. Harvard: Analytic Technologies.

Bresnen, M., \& Marshall, N. (2000). Partnering in construction: a critical review of issues, problems and dilemmas. Construction Management and Economics, 18(2), 229237. http://dx.doi.org/10.1080/014461900370852

Bryde, D., \& Leighton, D. (2009). Improving HEl Productivity and performance through project management implications from a benchmarking case study. Educational Management Administration \& Leadership, 37(5), 705721. http://dx.doi.org/10.1177/1741143209339649

Bryde, D. J. (2005). Methods for managing different perspectives of project success. British Journal of Management, 16(2), 119-131. http://dx.doi.org/10.1111/ j.1467-8551.2005.00438.x

Burns, T., \& Stalker, G. M. (1961). The management of innovation. London: Tavistock.

Campbell, C., Gibbs, A., Maimane, S., \& Yugi, N. (2008). Hearing community voices: grassroots perceptions of an intervention to support health volunteers in South Africa. Sahara J-Journal of Social Aspects of Hiv-Aids, 5(4), 162177. http://dx.doi.org/10.1080/17290376.2008.9724916

Campbell, C., Nair, Y., Maimane, S., \& Sibiya, Z. (2005). Building AIDS competent communities. AIDS Bulletin, 14(3), 14-19.

Chen, C. Y. (2011). Managing projects from a client perspective: the concept of the meetings-flow approach. International Journal of Project Management, 29(6): 671-686. http:// dx.doi.org/10.1016/j.jproman.2010.07.007

Cicmil, S., \& Marshall, D. (2005). Insights into collaboration at the project level: complexity, social interaction and procurement mechanisms. Building Research and Information, 33(6), 523-535. http://dx.doi. org/10.1080/09613210500288886

Ciomos, V. 1., Ciataras, D. M., \& Vlaicu, 1. (2011). Life cycle management for an investment project in Cluj-Salaj Area, Romania. Transylvanian Review of Administrative Sciences, (32E), 15-27.

Corder, G. D., Mclellan, B. C., \& Green, S. (2010) Incorporating sustainable development principles into minerals processing design and operation: SUSOP (R). Minerals Engineering, 23(3), 175-181. http://dx.doi. org/10.1016/j.mineng.2009.12.003

Couchman, P. K., \& Fulop, L. (2009). Examining partner experience in cross-sector collaborative projects focused on the commercialization of R\&D. InnovationManagement Policy \& Practice, 11(1): 85-103. http:// dx.doi.org/10.5172/impp.453.11.1.85

Courpasson, D., \& Clegg, S. (2006). Dissolving the iron cages? Tocqueville, Michels, bureaucracy and the perpetuation 
of elite power. Organization, 13(3): 319-343. http:// dx.doi.org/10.1177/1350508406063481

Cust, J. (2009). Using intermediate indicators: lessons for climate policy. Climate Policy, 9(5), 450-463. http:// dx.doi.org/10.3763/cpol.2009.0632

Davidson, P., Halcomb, E., Hickman, L., Phillips, J., \& Graham, B. (2006). Beyond the rhetoric: what do we mean by a 'model of care'? Australian Journal of Advanced Nursing, 23(3), 47-55. PMid:16568879.

Davies, A. (2009). Understanding local leadership in building the capacity of rural communities in Australia. Geographical Research, 47(4), 380-389. http://dx.doi. org/10.1111/j.1745-5871.2009.00586.x

De Bruijne, M., Van de Riet, O., De Haan, A., \& Koppenjan, J. (2010). Dealing with dilemma's: how can experiments contribute to a more sustainable mobility system? European Journal of Transport and Infrastructure Research, 10(3), 274-289.

Diodato, V. P. (1994). Dictionary of bibliometrics. New York: The Haworth Press.

Divakar, K. J. (2000). R\&D at Indian Organic Chemicals limited (10CL): from a service to an enterprise: a case study. $R$ \& D Management, 30(4), 341-348. http:// dx.doi.org/10.1111/1467-9310.00188

Dovers, S. R. (1995). A framework for scaling and framing policy problems in sustainability. Ecological Economics, 12 (2), 93-106. http://dx.doi.org/10.1016/09218009(94)00042-T

Dovers, S. R., \& Hadmer, J. W. (1992). Uncertainty, sustainability and change. Global Environmental Change, 2(4), 262-276. http://dx.doi.org/10.1016/09593780(92)90044-8

Drexler, J. A., \& Larson, E. W. (2000). Partnering: why project owner-contractor relationships change. Journal of Construction Engineering and ManagementAsce, 126(4), 293-297. http://dx.doi.org/10.1061/ (ASCE)0733-9364(2000)126:4(293)

Edwards, A., Sebba, J., \& Rickinsona, M. (2007). Working with users: some implications for educational research. British Educational Research Journal, 33(5), 647-661. http://dx.doi.org/10.1080/01411920701582199

Eglington, A., lsrael, R., \& Vartanov, R. (1998). Towards sustainable development for the Murmansk region. Ocean \& Coastal Management, 41(2-3), 257-271. http:// dx.doi.org/10.1016/S0964-5691(98)00068-4

Elkington, J. (2001) Canibais com garfo e faca: seria um sinal de progresso se um canibal utilizasse garfo e faca para comer? São Paulo: Makron Books.

Epp, H. T. (1995). Application of science to environmentalimpact assessment in boreal forest management: the Saskatchewan example. Water Air and Soil Pollution, 82(1-2), 179-188. http://dx.doi.org/10.1007/ BF01182832

Fellows, R., \& Liu, A. (2008). Impact of participants' values on construction sustainability. Proceedings of the Institution of Civil Engineers-Engineering Sustainability, 161(4), 219-227. http://dx.doi. org/10.1680/ensu.2008.161.4.219

Fernandez-Sanchez, G., \& Rodriguez-Lopez, F. (2010). A methodology to identify sustainability indicators in construction project management-application to infrastructure projects in Spain. Ecological
Indicators, 1O(6), 1193-1201. http://dx.doi.org/10.1016/j. ecolind.2010.04.009

Flyvbjerg, B. (2001). Making social science matter: why social inquiry fails and how it can succeed again. United Kingdom: Cambridge University Press. http://dx.doi. org/10.1017/CB09780511810503

Fourie, A., \& Brent. A. C. (2006). A project-based Mine Closure Model (MCM) for sustainable asset life Cycle Management. Journal of Cleaner Production, 14(1213), 1085-1095. http://dx.doi.org/10.1016/j. jclepro.2004.05.008

Frankel, A., Grillo, S. P., Pittman, M., Thomas, E. J., Horowitz, L., Page, M., et al. (2008). Revealing and resolving patient safety defects: the impact of leadership walkrounds on frontline caregiver assessments of patient safety. Health Services Research, 43(6), 2050-2066. PMid:18671751 PMCid:PMC2613998. http://dx.doi.org/10.1111/j.14756773.2008.00878.x

Gullick, D., Cairns, R., \& Pearson-Kirk, D. (2007). Application of partnering principles to a framework contract. Proceedings of the Institution of Civil EngineersMunicipal Engineer, 160(3), 127-133. http://dx.doi. org/10.1680/muen.2007.160.3.127

Haque, B. (2003). Lean engineering in the aerospace industry. Proceedings of the Institution of MechanicalEngineers Part B-Journal of Engineering Manufacture, 217(10), 14091420. http://dx.doi.org/10.1243/095440503322617180

Hartig, P. D., Hartig, J. H., Leshc, D. R., Lowriec, D. G., \& Weverd, G. H. (1996). Practical application of sustainable development in decision-making processes in the Great Lakes Basin. International Journal of Sustainable Development and World Ecology, 3(1), 31-46. http:// dx.doi.org/10.1080/13504509609469915

Henard, D. H., \& Szymanski, D. M. (2001). Why some new products are morel, successful than others. Journal of Marketing Research 38(3), 362-375. http://dx.doi. org/10.1509/jmkr.38.3.362.18861

Hirai, C., Uchida, Y., \& Fujinami, T. (2007). A knowledge management system for dynamic organizational knowledge circulation. International Journal of Information Technology \& Decision Making 6(3), 509522. http://dx.doi.org/10.1142/S0219622007002617

Hofstede, G. (1980). Culture's consequences: international differences in work-related values. Beverley Hills: Sage.

Houaiss, A., Villar, M. S., \& Franco, F. M. M. (2009). Dicionário Houaiss da língua portuguesa. Rio de Janeiro: Objetiva.

Hurt, M., \& Thomas, J. L. (2009). Building value through sustainable project management offices. Project Management Journal, 40(1), 55-72. http://dx.doi. org/10.1002/pmj.20095

Israr, S. M., \& Islam, A. (2006). Good governance and sustainability: a case study from Pakistan. International Journal of Health Planning and Management, 21(4), 313325. PMid:17175733. http://dx.doi.org/10.1002/ hpm.852

Jaglin, S., Repussard, C., \& Belbéoc'hc, A. (2011). Decentralisation and governance of drinking water services in small West African towns and villages (Benin, Mali, Senegal): the arduous process of building local governments. Canadian Journal of Development Studies-Revue Canadienne D Etudes Du Developpement, 32(2), 119-138. http://dx.doi.org/10.10 $80 / 02255189.2011 .596021$ 
Jiménez, J. B., \& Lorente, J. J. C. (2001). Environmental performance as an operations objective. International Journal of Operations \& Production Management, 21(12), 1553-1572. http://dx.doi. $\operatorname{org} / 10.1108 / 01443570110410900$

Joham, C., Metcalfe, M., \& Sastrowardoyo, S. (2009). Project conceptualization using pragmatic methods. International Journal of Project Management, 27(8), 787-794. http:// dx.doi.org/10.1016/j.jproman.2009.03.002

Jones, B. (2006). Trying harder: developing a new sustainable strategy for the UK. Natural Resources Forum, 30(2), 124-135. http://dx.doi.org/10.1111/ j.1477-8947.2006.00165.x

Kakabadse, N. K., Kakabadse, A. P., \& Summers, N. (2007). Effectiveness of Private Finance Initiatives (PFI): Study of private financing for the provision of capital assets for schools. Public Administration and Development, 27(1), 49-61. http://dx.doi.org/10.1002/ pad.433

Korkmaz, S., Riley, D., \& Horman, M. (2010). Piloting evaluation metrics for sustainable high-performance building project delivery. Journal of Construction Engineering and Management-Asce, 136(8), 877$885 . \quad \mathrm{http}: / / \mathrm{dx}$. doi.org/10.1061/(ASCE)CO.19437862.0000195

Kuper, M., Dionnet, M., Hammani, A., Bekka, Y., Garin, P., \& Bluemling, B. (2009). Supporting the shift from state water to community water: lessons from a social learning approach to designing joint irrigation projects in Morocco. Ecology and Society 14(1).

Larichev, O., Kochin, D., \& Kotnev, A. (2002) Decision support system for classification of a finite set of multicriteria alternatives. Decision Support Systems, 31(1), 13-21. http://dx.doi.org/10.1016/S0167-9236(01)00132-4

Lawrence, P. R., \& Lorsch, J. W. (1967). Organization and environment: managing differentiation and integration. Boston: Harward University.

Laws, D., \& Loeber, A. (2011). Sustainable development and professional practice. Proceedings of the Institution of Civil Engineers-Engineering Sustainability, 164(1), 2533. http://dx.doi.org/10.1680/ensu.2011.164.1.25

Lee, K. L., Wei, C. C., \& Lee, H. H. (2009). Reducing exposed copper on annular rings in a PCB factory through implementation of a Six Sigma project. Total Quality Management \& Business Excellence, 20(8), 863-876. http://dx.doi.org/10.1080/14783360903128322

Leurs, M. T. W., Mur-Veeman, I. M., Sar, R., Schaalma, H. P., \& Vries, N. K. (2008). Diagnosis of sustainable collaboration in health promotion: a case study. BMC Public Health 8, 382. PMid:18992132 PMCid:PMC2605462. http://dx.doi.org/10.1186/1471-2458-8-382

Liu, M. M. A. (2006). The framework underpinning conflicting keys in sustainability: harmony-in-transit. Property Management, 24(3), 219-231. http://dx.doi. org/10.1108/02637470610660129

Liu, M., Wu, X., Zhao, J. L., \& Zhu, L. (2010). Outsourcing of community source: identifying motivations and benefits. Journal of Global Information Management, 18(4), 3652. http://dx.doi.org/10.4018/jgim.2010100103

Madden, P. B. \& Morawski, J. D. (2011). The future of the canadian oil stands: engineering and project management advances. Energy \& Environment, 22(5), 579-596. http:// dx.doi.org/10.1260/0958-305X.22.5.579
Mahmoud-Jouini, S. B., \& Lenfle, S. (2010). Platform re-use lessons from the automotive industry. International Journal of Operations \& Production Management, 30(1), 98124. http://dx.doi.org/10.1108/01443571011012398

Marais, C., Van Wilgen, B. W., \& Stevens, D. (2004). The clearing of invasive alien plants in South Africa: a preliminary assessment of costs and progress. South African Journal of Science, 100(1), 97-103.

Mathews, L. B., Moodie, M. M., Simmons, A. M., \& Swinburn, B. A. (2010). The process evaluation of lt's Your Move!, an Australian adolescent community-based obesity prevention project. BMC Public Health, 10, 1-13. PMid:20670452 PMCid:PMC2920280. http://dx.doi. org/10.1186/1471-2458-10-448

McNulty, C. A. M., Freeman, E., Oliver, l., Ford-Young, W., \& Randall, S. (2008). Strategies used to increase chlamydia screening in general practice: a qualitative study. Public Health, 122(9), 845-856. PMid:18620715. http://dx.doi. org/10.1016/j.puhe.2007.10.009

Mewaldt, A. (2010). Forming the civil sector in transformation countries: localisation processes of civil society organisations and across-the-sectors development partnership. Gruppendynamik Und Organisationsberatung, 41(3), 235-254. http://dx.doi. org/10.1007/s11612-010-0117-4

Migliarese, P., \& Paolucci, E. (1995). Improved communications and collaborations among tasks induced by groupware. Decision Support Systems, 14(3), 237-250. http://dx.doi. org/10.1016/0167-9236(94)00019-0

Mohammadi, S. M., Mohammadi, S. F., Hedges, J. R., Zohrabi, M., \& Ameli, 0. (2007). Introduction of a quality improvement program in a children's hospital in Tehran: design, implementation, evaluation and lessons learned. International Journal for Quality in Health Care, 19(4), 237-243. PMid:17573405. http://dx.doi. org/10.1093/intqhe/mzm021

Molenaar, K. R., Sobin, N., \& Antillón, E. I. (2010). A synthesis of best-value procurement practices for sustainable design-build projects in the public sector. Journal of Green Building, 5(4), 148-157. http://dx.doi. org/10.3992/jgb.5.4.148

Mulder, J., \& Brent, A. C. (2006). Selection of sustainable rural agriculture projects in South Africa: case studies in the LandCare programme. Journal of Sustainable Agriculture, 28(2), 55-84. http://dx.doi.org/10.1300/ J064v28n02_06

Nair, Y., \& Campbell, C. (2008). Building partnerships to support community-led HIV/AIDS management: a case study from rural South Africa. Ajar-African Journal of Aids Research, 7(1), 45-53. http://dx.doi.org/10.2989/ AJAR.2008.7.1.6.434

Nath, T. K., \& Inoue, M. (2010). Impacts of participatory forestry on livelihoods of ethnic people: experience from Bangladesh. Society \& Natural Resources, 23(11), 10931107. http://dx.doi.org/10.1080/08941920802653521

Naylor, P. J., Wharf-Higgins, J., Blair, L., Green, L., \& O'Connor, B. (2002). Evaluating the participatory process in a community-based heart health project. Social Science \& Medicine, 55(7), 1173-1187. http://dx.doi. org/10.1016/S0277-9536(01)00247-7

Neely, A. (2005). The evolution of performance measurement research: developments in the last decade and a research agenda for the next. International Journal of Operations 
\& Production Management, 25(18), 1264-1277. http:// dx.doi.org/10.1108/01443570510633648

Nielsen, K. R. (2006). Risk management: lessons from six continents. Journal of Management in Engineering, 22(2), 61-67. http://dx.doi.org/10.1061/ (ASCE)0742-597X(2006)22:2(61)

Nijland, H. J. (2005). Sustainable Development of Floodplains (SDF) project. Environmental Science \& Policy, 8(3), 245252. http://dx.doi.org/10.1016/j.envsci.2005.03.002

Nyamadi, B., \& Yokogawa, H. (1997). Project management turnover to farmers in Ghana: results of a farmer survey. Journal of the Faculty of Agriculture Kyushu University, 42(1-2), 249-264.

Ohno, T. (1988). The Toyota production system: beyond large scale production. Portland: Productivity Press.

Olney, C. A., Backus, J. E. B., \& Klein, L. J. (2010). Characteristics of project management at institutions sponsoring National Library of Medicine Medline Plus go local. Journal of the Medical Library Association, 98(1), 6572. PMid:20098657 PMCid:PMC2801972. http://dx.doi. org/10.3163/1536-5050.98.1.018

Ottmann, G., \& Street, A. F. (2007). Ten lessons for developing a health information website. Australian Health Review, 31(4), 523-526. PMid:17973609. http:// dx.doi.org/10.1071/AH070523

Pacheco-de-Almeida, G., \& Zemsky, P. (2007). The timing of resource development and sustainable competitive advantage. Management Science, 53(4), 651-666. http:// dx.doi.org/10.1287/mnsc.1060.0684

Pavez, l., Gonzalez, V., \& Alarcón, L. F. (2010). Improving the effectiveness of new construction management philosophies using the integral theory. Revista de la Construccion, 9(1), 26-38.

Petter, S., Khazanchi, D., \& Murphy, J. D. (2010). A design science based evaluation framework for patterns. Data Base for Advances in Information Systems, 41(3), 9-26. http://dx.doi.org/10.1145/1851175.1851177

Porter, M. E. (1985). Competitive advantage. New York: Free Press.

Project Management Institute - PMl. (2008) A guide to the project management body of knowledge (PMBoK) (4. ed.). Pennsylvania: Project Management Institute.

Prahalad, C., \& Hamel, G. (1990). The core competence of the organization. Harvard Business Review, 68(3), 79-91.

Pulaski, M. H., \& Horman, M. J. (2005). Continuous value enhancement process. Journal of Construction Engineering and Management-Asce, 131(12), 12741282 http://dx.doi.org/10.1061/(ASCE)07339364(2005)131:12(1274)

Pulaski, M. H., Horman, M. J., \& Chandra, M. J. (2006). Case study validation of the continuous value enhancement process. Journal of Green Building, 1(3), 169-182. http://dx.doi.org/10.3992/jgb.1.3.169

Quante, R. (2010). Building measures in order to create value add in real estate asset management - scope of services and key figures for optimization of real estate investments. Bauingenieur, 85, 71-81.

Raven, R., Jolivet, E., Mourik, R. M., \& Feenstra, C. F. J. (2009). ESTEEM: managing societal acceptance in new energy projects A toolbox method for project managers. Technological Forecasting and Social Change, 76(7), 963977. http://dx.doi.org/10.1016/j.techfore.2009.02.005
Read, H. W., \& Napier, L. G. D. (1994). Project-management, and the design of shaft-sinking projects. Journal of the South African Institute of Mining and Metallurgy, 94(7), 147-171.

Reed, J. (2005). Using action research in nursing practice with older people: democratizing knowledge. Journal of Clinical Nursing, 14(5), 594-600. PMid:15840074. http://dx.doi.org/10.1111/j.1365-2702.2005.01110.x

Robichaud, L. B., \& Anantatmula, V. S. (2011). Greening project management practices for sustainable construction. Journal of Management in Engineering, 271), 48-57. http://dx.doi.org/10.1061/(ASCE)ME.1943-5479.0000030

Salem, 0., \& Mohanty, S. (2008). Project management practices and information technology research. Journal of Construction Engineering and ManagementAsce, 134(7), 501-508. http://dx.doi.org/10.1061/ (ASCE)0733-9364(2008)134:7(501)

Sanderson, J., \& Cox, A. (2008). The challenges of supply strategy selection in a project environment: evidence from UK naval shipbuilding. Supply Chain Managementan International Journal, 13(1), 16-25. http://dx.doi. org/10.1108/13598540810850283

Schildt, H. (2002). Sitkis: software for bibliometric data management and analysis. Helsinki: Institute of Strategy and International Business.

Schuman, C. A., \& Brent, A. C. (2005). Asset life cycle management: towards improving physical asset performance in the process industry. International Journal of Operations \& Production Management, 25, (5-6), 566579. http://dx.doi.org/10.1108/01443570510599728

Seitz, D., Kerlen, C., Steg, H., \& Schott, D. (2005). Results of an evaluation of company organizational development. Zeitschrift für Evaluation, (2), 245-269.

Sell, J., Koellner, T., Weber, O., Pedroni, L., \& Scholz, R. W. (2006). Decision criteria of European and Latin American market actors for tropical forestry projects providing environmental services. Ecological Economics, 58(1), 1736. http://dx.doi.org/10.1016/j.ecolecon.2005.05.020

Sengoku, S., Yoda, T., \& Seki, A. (2011). Assessment of pharmaceutical research and development productivity with a novel net present value-based project database. Drug Information Journal, 5(2), 175-185.

Shore, B., \& Cross, B. J. (2004). Maintaining funding in largescale international science projects. International Journal of Technology Management, 27(4), 417-430. http:// dx.doi.org/10.1504/1JTM.2004.004106

Sieli, E. M. (1991). Managing a project as a process. At\&T Technical Journal, 70(2), 33-39. http://dx.doi. org/10.1002/j.1538-7305.1991.tb00343.x

Smeda, R. (2001). Implementation of business process innovations: an agenda for research and action. International Journal of Technology Management, 22(13), 1-12. http://dx.doi.org/10.1504/IJTM.2001.002951

Steiner, J. L., Sadler, E. J., Chen, J. S., Wilson, G., James, D., Vandenberg, B., Ross, J., Oster, T., \& Cole, K. (2008). Sustaining the earth's watersheds-agricultural research data system: overview of development and challenges. Journal of Soil and Water Conservation, 63(6), 569-576. http://dx.doi.org/10.2489/jswc.63.6.569

Stevens, B., \& Peikes, D. (2006). When the funding stops: Do grantees of the local initiative funding partners program sustain themselves? Evaluation and Program 
Planning, 29(2), 153-161. http://dx.doi.org/10.1016/j. evalprogplan.2006.01.009

Thoma, K., Baker, P. A., \& Allender, E. B. (1993). Design methods for the development of waste-water land disposal systems. Water Science and Technology, 27(1), 77-86.

Thomas, A. J. (2007). Creating sustainable small to medium enterprises through technological innovation. Proceedings of the Institution of Mechanical Engineers Part B-Journal of Engineering Manufacture, 221(3), 513528. http://dx.doi.org/10.1243/09544054JEM524

Thomson, C. S., El-Haram, M. A., \& Emmanuel, R. (2011). Mapping sustainability assessment with the project life cycle. Proceedings of the Institution of Civil EngineersEngineering Sustainability, 164(2), 143-157. http:// dx.doi.org/10.1680/ensu.2011.164.2.143

Tsai, W. H., Lin, S. J., Liub, J. Y., Lina, W. R., \& Lee, K. C. (2011). Incorporating life cycle assessments into building project decision-making: an energy consumption and $\mathrm{CO}_{2}$ emission perspective. Energy, 36(5), 3022-3029. http://dx.doi.org/10.1016/j.energy.2011.02.046

Turlea, C., Roman, T. D., \& Constantinescu, D. G. (2010). The project management and the need for sustainable development. Metalurgia International, 15(3), 121-125.

Ustinovichius, L., \& Kochin, D. (2003). Verbal analysis of the investment risk in construction. Journal of Business Economics and Management, 4(4), 228-234.

Ustinovichius, L., Shevchenko, G., Barvidas, A., Ashikhmin, 1. V., \& Kochin, D. (2010). Feasibility of verbal analysis application to solving the problems of investment in construction. Automation in Construction, 19(3), 375384. http://dx.doi.org/10.1016/j.autcon.2009.12.004

Van Berkel, R. (2010). Evolution and diversification of National Cleaner Production Centres (NCPCs). Journal of Environmental Management, 91(7), 15561565. PMid:20346575. http://dx.doi.org/10.1016/j. jenvman.2010.02.032

Veas, L., \& Pradena, M. (2008). The integral project manager in the construction industry. Revista De La Construccion, 7(2), 47-55.

Wasserman, S., \& Faust, K. (1994). Social network analysis:methods and applications. Cambridge: Cambridge University Press. http://dx.doi.org/10.1017/ CB09780511815478

Weerasinghe, G., Soundararajan, K., \& Ruwanpura, J. (2007). Leed-pdri framework for pre-project planning of sustainable building projects. Journal of Green Building, 2(3), 123-143. http://dx.doi.org/10.3992/ jgb.2.3.123
Wesselink, R., \& Wals, A. E. J. (2011). Developing competence profiles for educators in environmental education organisations in the Netherlands. Environmental Education Research, 17(1), 69-90. http://dx.doi. org/10.1080/13504621003637037

Williams, T. (1999). The need for new paradigms for complex projects. International Journal of Project Management, 17(5), 269-273. http://dx.doi.org/10.1016/ S0263-7863(98)00047-7

Williams, T., Ackermann, F., \& Eden, C. (2003). Structuring a delay and disruption claim: an application of causemapping and system dynamics. European Journal of Operational Research, 148(1), 192-204. http://dx.doi. org/10.1016/S0377-2217(02)00372-7

Windel, l. (2005). Learning to live healthy: health management in schools. Health promotion in schools with particular consideration of socially disadvantaged students. Gesundheitswesen, 67(2), 137-140. PMid:15747203. http://dx.doi.org/10.1055/s-2005-857890

Womack, J. P., Jones, D. T., \& Roos, D. (1990). The machine that changed the world. New York: Rawson Associates.

Wright, S. (2000). Project management of Greenwich peninsula. Proceedings of the Institution of Civil Engineers-Civil Engineering, 138, 19-23. http://dx.doi. org/10.1680/cien.2000.138.5.19

Wu, P., \& Low, S. P. (2010). Project Management and Green Buildings: lessons from the Rating Systems. Journal of Professional Issues in Engineering Education and Practice, 136(2), 64-70. http://dx.doi.org/10.1061/ (ASCE)El.1943-5541.0000006

Zahay, D., Griffin, A., \& Fredericks, E. (2011). Information use in new product development: an initial exploratory empirical investigation in the chemical industry. Journal of Product Innovation Management, 28(4), 485-502. http://dx.doi.org/10.1111/j.1540-5885.2011.00821.x

Zhang, X., Fu, Z., Wengui, C., Dong, T., \& Jian, Z. (2009). Applying evolutionary prototyping model in developing FIDSS: an intelligent decision support system for fish disease/health management. Expert Systems with Applications, 36(2), 3901-3913. http://dx.doi. org/10.1016/j.eswa.2008.02.049

\section{Agradecimentos}

Agradecemos aos avaliadores do trabalho, que muito contribuíram para a pesquisa com os seus comentários e sugestões. Agradecemos também à Capes e ao CNPq pelo suporte ao projeto de pesquisa. 


\section{Sustainability and management of projects: a bibliometric study}

\section{Abstract}

Projects conducted in firms and organizations have immediate impacts as well as long-term consequences. The objective of this paper is to understand the treatment of sustainability issues in the literature on project management. The methodology incorporates bibliometric techniques, network analysis and content analysis. The results indicate that the publications are dispersed among many authors and journals. The themes relate to issues such as stakeholders, innovation and product development, with an emphasis on publications in the area of construction. There is a tendency, especially after 2005, toward articles focused on the economic pillar and on increasing participation in social and environmental approaches. In just the last two years, studies with a focus on the triple constraint - social, environmental and economic - have increased considerably. There are significant opportunities for research because the publications are far from exhaustive on this complex subject.

\section{Keywords}

Project management. Sustainability. Bibliometric study. 